\title{
Towards A Model-based Prognostics Methodology for Electrolytic Capacitors: A Case Study Based on Electrical Overstress Accelerated Aging
}

\author{
José R. Celaya ${ }^{1}$, Chetan S. Kulkarni ${ }^{2}$, Gautam Biswas ${ }^{3}$, and Kai Goebel ${ }^{4}$ \\ ${ }^{1}$ SGT Inc. NASA Ames Research Center, Moffett Field, CA, 94035, USA \\ jose.r.celaya@nasa.gov \\ ${ }^{2,3}$ Vanderbilt University, Nashville, TN, 37235, USA \\ chetan.kulkarni@vanderbilt.edu \\ biswas@eecsmail.vuse.vanderbilt.edu \\ ${ }^{5}$ NASA Ames Research Center, Moffett Field, CA, 94035, USA \\ kai.goebel@nasa.gov
}

\begin{abstract}
This paper presents a model-driven methodology for predicting the remaining useful life of electrolytic capacitors. This methodology adopts a Kalman filter approach in conjunction with an empirical state-based degradation model to predict the degradation of capacitor parameters through the life of the capacitor. Electrolytic capacitors are important components of systems that range from power supplies on critical avionics equipment to power drivers for electro-mechanical actuators. These devices are known for their comparatively low reliability and given their critical role in the system, they are good candidates for component level prognostics and health management. Prognostics provides a way to assess remaining useful life of a capacitor based on its current state of health and its anticipated future usage and operational conditions. This paper proposes and empirical degradation model and discusses experimental results for an accelerated aging test performed on a set of identical capacitors subjected to electrical stress. The data forms the basis for developing the Kalman-filter based remaining life prediction algorithm.
\end{abstract}

\section{INTRODUCTION}

This paper proposes the use of a model based prognostics approach for electrolytic capacitors. Electrolytic capacitors are critical components in electronic systems in aeronautics applications and in other domains. This type of capacitors are known to have lower reliability than other electronic com-

José R. Celaya et.al. This is an open-access article distributed under the terms of the Creative Commons Attribution 3.0 United States License, which permits unrestricted use, distribution, and reproduction in any medium, provided the original author and source are credited. ponents that are used in power supplies of avionics equipment and electrical drivers of electro-mechanical actuators of aircraft control surfaces. The field of prognostics for electronics is concerned with the prediction of remaining useful life (RUL) of the components and systems. This notion of condition-based health assessment leverages the knowledge of the device physics in order to model the degradation process, which is then used to predict remaining useful life as a function of current state of health and anticipated future operational and environmental conditions.

The prognostics methodology presented here, is based on the Bayesian tracking framework and a dynamic degradation model developed empirically from electrical overstress accelerated aging tests. A validation methodology is presented to assess the validity of the method using available run-to-failure data. The novelty of the approach consists on its ability to periodically estimate remaining useful life. This estimation process is condition-based in the sense that periodic measurements of the component under consideration are used in the estimation process. The contributions of this work are a dynamic degradation model and a model-based prognostics methodology for electrolytic capacitors. We present results for estimation of remaining useful life for five test cases. Predictions are made at several points in time during the life of the capacitors. Performance metrics like median relative accuracy and the $\alpha-\lambda$ metric demonstrate the effectiveness of our approach.

\subsection{Motivation}

The development of prognostics methodologies for electronic systems has become more important as more electrical sys- 
tems are being used to replace traditional systems in several applications in the aeronautics, maritime, and automotive fields. The development of prognostics methods for electronics presents several challenges due to the great variety of components used in a system, the continual evolution of new electronics technologies, and a general lack of understanding of how electronics fail. Traditional reliability techniques in electronics tend to focus on understanding the time to failure for a batch of components of the same type, by running multiple experiments and making statistical estimates from the accumulated time to failure data. Recently, there has been a push to understand, in more depth, how faults progress as a function of time, loading, and environmental conditions. Furthermore, just until recently, it was believed that there were no precursor to failure indications for electronic components and systems. That is now understood to be incorrect, since electronic systems, much like mechanical systems, undergo a measurable wear process from which one can derive features that can be used to provide early warnings to failure. The indications of degradation caused by the wear can be detected fairly early, and by modeling the process, one can potentially predict the remaining useful life as a function of future use and environmental conditions.

Avionic systems perform critical functions on aircraft, greatly escalating the ramification of an in-flight malfunction (Bhatti \& Ochieng, 2007; Kulkarni et al., 2009). Avionic systems combine physical processes, computational hardware and software. These elements present unique challenges for fault detection and isolation. A systematic analysis of these elements and their interaction is very important for the assessment of aircraft safety and to avoid catastrophic failures during flight.

Power supplies are critical components of modern avionic systems. In navigation systems, for instance, degradation and faults in the DC-DC converter power source unit propagate to the global positioning system (GPS) and other navigation subsystems, affecting the overall operations of the aircraft. Capacitors and power metal oxide field effect transistors (MOSFETs) are the two major components that cause performance degradation and failures in DC-DC converters (Kulkarni, Biswas, Bharadwaj, \& Kim, 2010). Some of the more prevalent fault effects, such as a ripple voltage surge at the power supply output, can cause glitches in the GPS position and velocity output, and this in turn, if not corrected, can propagate and distort the navigation process.

Electrical motors are an essential element in electromechanical actuators systems that are being used to replace hydro-mechanical actuation in control surfaces of future generation aircrafts. Capacitors are used as filtering elements on power electronic systems, particularly for motor drivers. Electrical power drivers for motors require capacitors to filter the rail voltage for the $\mathrm{H}$-bridges that provide bidirectional current flow to the windings of electrical motors. These capacitors help to ensure that the heavy dynamic loads generated by the motors do not perturb the upstream power distribution system. A failure in a rail voltage filter capacitor will have effects on the power distribution system and on performance of the motor, which will have cascading effects on the actuation process.

Low reliability and their criticality in avionics systems make electrolytic capacitors important candidates for focused health management solutions. In addition to this, degradation at component level could lead to cascading faults at subsystem and system levels. In order to mitigate the effects of capacitor failures in critical to safety systems, we introduce here, a condition-based prognostics methodology. This methodology develops the ability to identify degradation effects and to estimate the remaining life of the components progressively in time. This method will further allow for prognostics-based decision making for condition-based maintenance scheduling of the system or for implementation of mitigation strategies in case of contingencies during operation. In the next section we discuss briefly earlier work on capacitors at both component and system level.

\subsection{Previous work}

In earlier work, we studied the degradation of capacitors under nominal operation (Kulkarni, Biswas, Koutsoukos, Goebel, \& Celaya, 2010b). The capacitors were components in a DC-DC converter, and their degradation was monitored every 100-120 hours of operation. Data was collected to determine the change in equivalent series resistance (ESR) and capacitance. An Arrhenius inspired ESR degradation model for time to failure computation was presented in Kulkarni, Biswas, Koutsoukos, Goebel, and Celaya (2010a). The data collected during the monitoring steps were used to compute the parameters of the model as well as for model validation.

In subsequent experimental work, we studied accelerated degradation in capacitors subjected to high charge/discharge cycles at a constant frequency (Kulkarni, Biswas, Koutsoukos, Celaya, \& Goebel, 2010). A preliminary approach to computing RUL of electrolytic capacitors was presented in Celaya et al. (2011b).

In Kulkarni, Celaya, Goebel, and Biswas (2012b) we studied capacitor degradation under thermal overstress conditions. A physics-based degradation model, based on physics' first principles, was derived for the electrolytic capacitors and the derived model was employed in making RUL estimations based on a Bayesian tracking methodology (Kulkarni, Celaya, Goebel, \& Biswas, 2012c, 2012a).

This paper here builds upon the initial studies presented in Celaya et al. (2011a) and Celaya et al. (2012). 


\subsection{Other related work and current art in capacitor prog- nostics}

The output filter capacitor has been identified as one of the elements of a switched mode power supply that fails more frequently and has a critical impact on performance (Goodman et al., 2007; Judkins et al., 2007; Orsagh et al., 2005). A prognostics and health management approach for power supplies of avionics systems is presented in Orsagh et al. (2005). Results from accelerated aging of the complete supply have been discussed in terms of output capacitor and power MOSFET failures; but there is no modeling of the degradation process or RUL prediction for the power supply. Other approaches for prognostics for switched mode power supplies are presented in Goodman et al. (2007) and Judkins et al. (2007). The output ripple voltage and leakage current are presented as a function of time and degradation of the capacitor, but no details of the degradation process modeling, fault detection, and RUL prediction algorithms were presented.

A health management approach for multilayer ceramic capacitors is presented in Nie et al. (2007). This approach focuses on the temperature-humidity bias accelerated test to replicate failures. A method based on Mahalanobis distance is used to detect abnormalities in the test data; there is no prediction of RUL. A data driven prognostics algorithm for multilayer ceramic capacitors is presented in $\mathrm{Gu}$ et al. (2008). This method uses data from accelerated aging test to detect potential failures and to make an estimation of time of failure occurrence.

The approaches discussed above address fault detection and diagnostics methods using data-driven approaches. Our work focuses in prognostics, which is the natural progression from diagnostics. In addition, our methodology is based on dynamic degradation models used as part of a model-based prognostics framework.

\section{Prognostics Methodology}

A model-based prognostics methodology for electrolytic capacitors is presented in this section. This methodology relies on accelerated aging experiments to identify degradation behavior and to create time dependent degradation models. The process followed in the proposed methodology is presented in the block diagram in Figure 1 and described next.

Accelerated Aging: The methodology is based on results from an accelerated life test on real electrolytic capacitors. This test applies electrical overstress to commercial, off the shelf capacitors, in order to observe and record the degradation process and identify performance conditions in the neighborhood of the failure criteria in a considerably reduced time frame. A total of 6 test devices are used for this accelerated aging study. Electrochemical-impedance spectroscopy (EIS) is used periodically during the accelerated ag- ing test to characterize the frequency response of the capacitor's impedance. Several measurements are made through the aging process, starting from measurements made under pristine condition all the way through to complete failure.

System Identification: A lumped-parameter model $\left(\mathcal{M}_{1}\right)$ of the non-ideal capacitor impedance is assumed. This impedance model includes a capacitance element and a parasitic equivalent series resistance element. The EIS measurements along with the impedance model structure are used in a systems identification setting to estimate the model parameters. This is done for all the EIS measurements at different points in time during the aging experiment, resulting in timedependent capacitance and ESR measurement trajectories reflecting capacitor degradation.

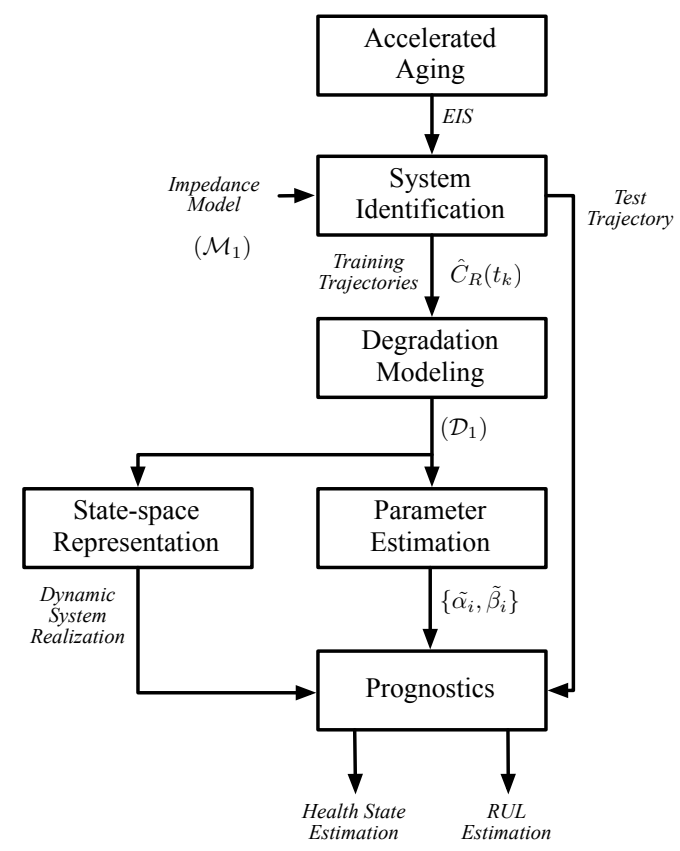

Figure 1. Methodology for the development of the capacitor prognostics approach.

Degradation Modeling: We present here an empirical degradation model that is based on the observed degradation process during the accelerated life test $\left(\hat{C}_{R}\left(t_{k}\right)\right)$. The objective of the modeling is to generate a parametrized model $\left(\mathcal{D}_{1}\right)$ of the time-dependent capacitance degradation as generated by the system identification step. A similar degradation model can be generated for ESR but it is not discussed in this work.

Parameter Estimation: A traditional training+test method is used to derive and validate the capacitor degradation model. The parameters of the degradation model are estimated using nonlinear least-squares regression. The quality of the parameter estimation results is satisfactory as to assume the estimated parameters $\left(\tilde{\alpha}_{i}, \tilde{\beta}_{i}\right)$ are static (not time-dependent) during the prognostics process described next. 
Prognostics: A Bayesian framework is employed to estimate (track) the state of health of the capacitor based on measurement updates for key capacitor parameters. The Kalman filter algorithm is used to track the state of health and the degradation model is used to make health state forecasts to be used in computation of remaining useful life once no further measurements are available.

\section{ACCELERATED AGING EXPERIMENTS}

Accelerated life test methods are often used in prognostics research as a way to assess the effects of the degradation process through time. It also allows for the identification and study of different failure mechanisms and their relationships with different observable signals and parameters. In this section we present a brief description of the accelerated electrical overstress experiment for studying capacitor degradation. We provide insights into the physical interpretation of the underlying degradation process under the electrical stress condition. Finally, we present a discussion on how the systems identification approach is used to compute the precursor to failure features based electrochemical impedance spectroscopy measurements obtained during the aging experiment.

\subsection{Experimental Setup}

Since the objective of this experiment is the study of the effects of high voltage on degradation of the capacitors, the capacitors were subjected to high voltage stress through an external power source using custom developed hardware. The voltage overstress is applied to the capacitors as a square waveform in order to subject the capacitor to continuous charge and discharge cycles.

At the beginning of the accelerated aging, the capacitors charge and discharge simultaneously; as time progresses and the capacitors degrade at different rates, the charge and discharge times vary for each capacitor. Even though all the capacitors under test are subjected to the same loading and operating conditions, their ESR and capacitance values change differently. We therefore monitor charging and discharging of each capacitor under test and measure the input and output voltages of the capacitor as well as the load current. Figure 2 illustrates the electrical overstress experiment's electrical circuit. A function generator is used to generate a square waveform, which is then amplified to the desired amplitude to be applied to the unit under test (UUT). A resistive load is used in series with the capacitor in order to emulate the loading side of a first order passive filter. Additional details on the accelerated aging system are presented in (Kulkarni, Biswas, Koutsoukos, Celaya, \& Goebel, 2010).

For the experiment reported in this paper, a set of six capacitors was considered. Electrolytic capacitors of $2200 \mu \mathrm{F}$ capacitance, with a maximum rated voltage of $10 \mathrm{~V}$, maxi- mum current rating of $1 A$ and maximum operating temperature of $105^{\circ} \mathrm{C}$ were used for the study. The electrolytic capacitors under test were characterized by EIS measurements before the start of the experiment and at different stages during the experiment execution. The experiment was conducted at room temperature.

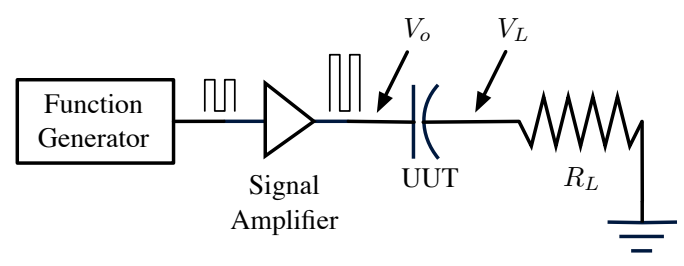

Figure 2. Block diagram of the experimental setup.

The EIS measurements were recorded every 8-10 hours of the total 180 plus hours of accelerated aging time in order to capture the degradation phenomenon in the ESR and capacitance values. During each measurement, the voltage source was shut down, the capacitors were discharged completely and then the EIS characterization procedure was carried out. This was done for all the six capacitors under test. A picture of the actual experiment setup is presented in Figure 3. For further details regarding the aging experiment, results and analysis of the measured data refer to (Kulkarni, Biswas, Koutsoukos, Celaya, \& Goebel, 2010; Celaya et al., 2011b).

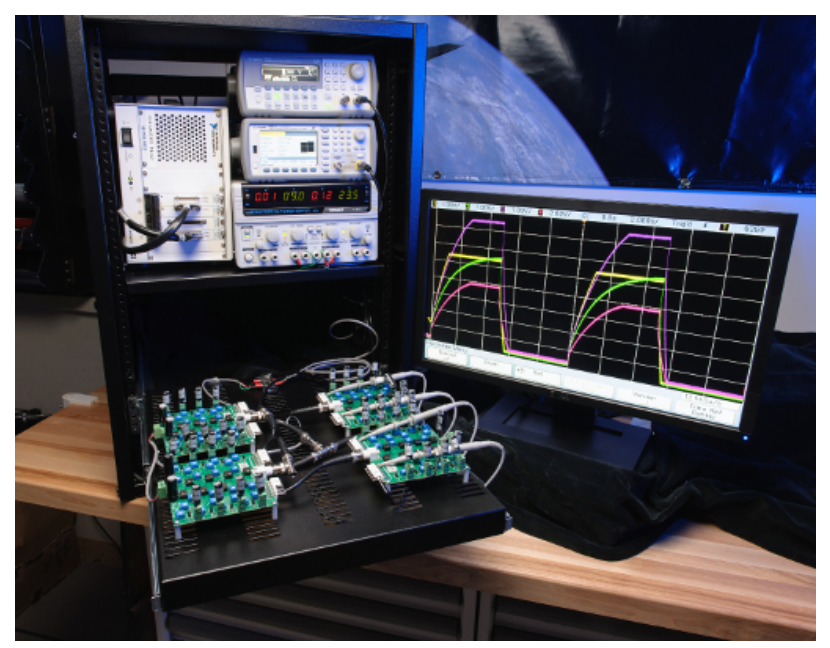

Figure 3. Electrical overstress aging experiment.

\subsection{Physical interpretation of the degradation process}

There are several factors that cause electrolytic capacitors to fail. Continuous degradation, in the form of gradual loss of functionality over a period of time, eventually results in the failure of the component. A complete loss of function is termed a catastrophic failure. Typically, this results in a short or open circuit in the capacitor. 
In this work, we study the degradation of electrolytic capacitors operating under electrical stress, where $V_{\text {applied }} \geq$ $V_{\text {rated. }}$. During the charge/discharge process, the capacitors degrade over time. A study of the literature indicates that the degradation can be primarily attributed to electrolyte evaporation, leakage current and increased internal pressure due to gas released during chemical reactions (International Standard IEC 60384-4-1: Fixed capacitors for use in electronic equipment, 2007; MIL-C-62F, 2008; Kulkarni, Biswas, Koutsoukos, Goebel, \& Celaya, 2010a). Our primary focus in this work is the study of the degradation due to electrolyte evaporation.

Figure 4 shows the structure of an electrolytic capacitor in detail. An ideal capacitor, while charging, would offer no resistance to the flow of current at its leads. However, the electrolyte that fills the space between the plates and the electrodes produces a small series resistance known as ESR. The quantitative changes in the ESR and capacitance values typify the current health state of the device, and represent the two primary precursors to failure. ESR and capacitance values were calculated after characterizing the capacitors at regular intervals. The heat generated due to current flow through the capacitor's internal resistance, ESR, during the operation of the capacitor, causes an increase in the internal temperature which also increases the rate of electrolyte evaporation. Decrease in electrolyte volume due to evaporation leads to further increases in ESR and decrease in the effective oxide surface area, resulting in capacitance decrease. The literature on capacitor degradation shows a direct relationship between electrolyte decrease with increase in ESR and decrease in capacitance value of the capacitor (Kulkarni, Biswas, Koutsoukos, Goebel, \& Celaya, 2010b).

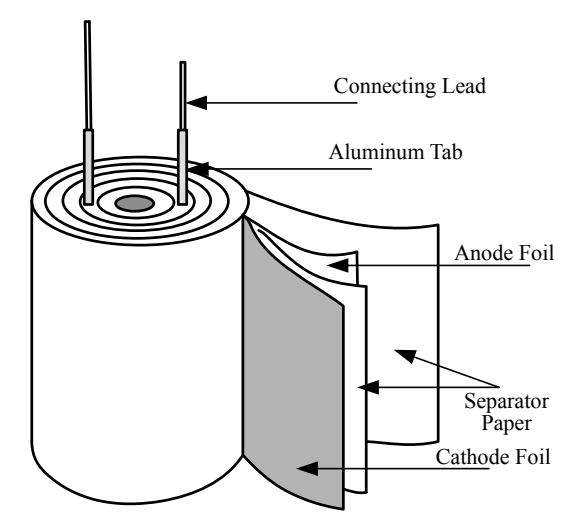

Figure 4. Electrolytic capacitor structure.

\subsection{System identification for non-ideal capacitor model}

ESR and capacitance values are estimated by using a system identification approach over a lumped parameter model $\left(\mathcal{M}_{1}\right)$ consisting of the capacitance and the ESR in series as shown in Figure 5. It should be noted that the lumped-parameter model used to estimate ESR and capacitance offline, is not the degradation evolution model to be used on the online elements of the prognostics algorithm; it only allows us to estimate parameters, which provide indications of the degradation process through time. The impedance's frequency response is used for the system identification. Parameters such as ESR and capacitance are more difficult to estimate from the in-situ measurements of voltage and current partially available through the accelerated aging experiments. This can be done with a recursive estimation approach but it is not discussed in this paper.

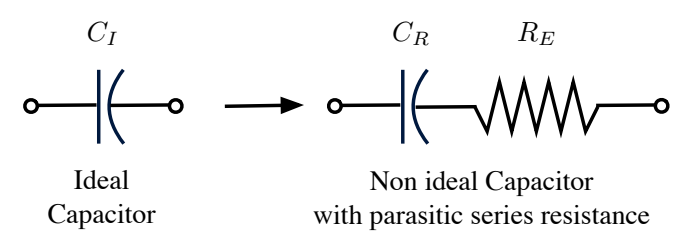

Figure 5. Lumped parameter model $\left(\mathcal{M}_{1}\right)$ for a non-ideal capacitor.

The ESR and capacitance values were estimated from the capacitor electrochemical impedance frequency response measured using an SP-150 Biologic instrument. The ideal capacitor has complex impedance $Z_{I}=1 / s C_{I}$, where $C_{I}$ is the ideal capacitance value. The complex impedance of model $\mathcal{M}_{1}$ is given by

$$
\mathcal{M}_{1}: Z=R_{E}+\frac{1}{s C_{R}},
$$

where $R_{E}$ is the equivalent series resistance and $C_{R}$ is the real capacitance.

Electrochemical impedance spectroscopy measurements are available to characterize the electrical performance of the capacitor throughout the aging experiment. Figure 6 shows Nyquist plots of the impedance measurements for capacitor \#1 at pristine condition and after accelerated aging times 71, 161 and 194 hours. The degradation can be observed as the Nyquist plot shifts to the right as a function of aging time due to increase in $R_{E}$. These measurement are then used to estimate the parameters of the impedance model $\mathcal{M}_{1}$ from Eq. (1). The parameter estimation performed using the EIS instrument software (EC-Lab $\left.{ }^{\circledR}\right)$. This is basically and optimization approach using an objective function defined as the aggregate of the squared error for all the frequencies where there is an impedance measurement from EIS. The error is computed based on the difference in magnitude of the model and the measured impedance. The optimization is set up to minimize the objective function by finding optimal values $C_{R}{ }^{*}$ and $R_{E}{ }^{*}$ for the $\mathcal{M}_{1}$ model.

This parameter estimation is performed every time an EIS measurement is made, resulting in values of $C_{R}$ and $R_{E}$ at different points in time through the aging of the components 
$\left(C_{R}\left(t_{k}\right)\right.$ and $\left.R_{E}\left(t_{k}\right)\right)$. The average pristine condition ESR was measured to be $0.056 \mathrm{~m} \Omega$ and the average pristine condition capacitance was measured to be $2123 \mu \mathrm{F}$ for the set of capacitors under test.

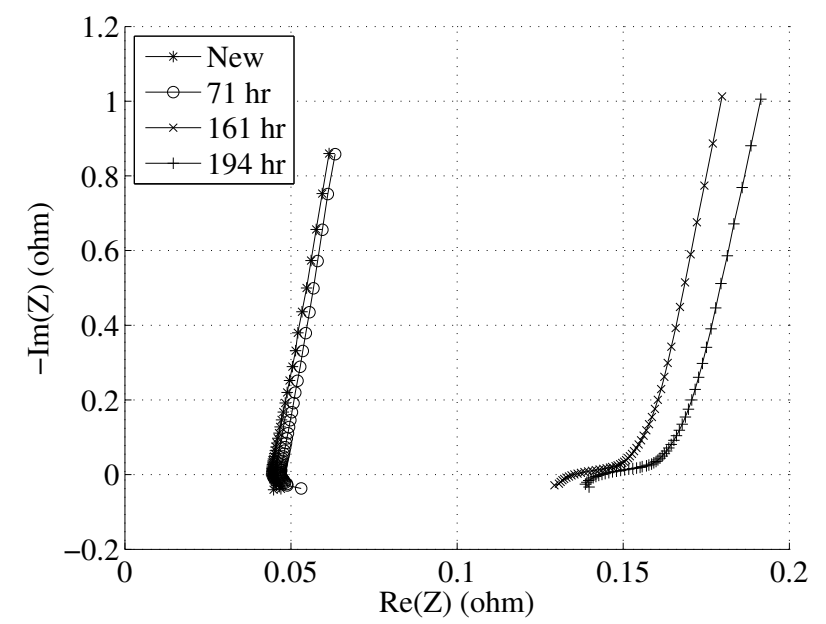

Figure 6. Electrochemical impedance measurements at different aging times.

Figure 7 shows the percentage increase in the ESR value for all the six capacitors under test over the aging time. Similarly, Figure 8 shows the percentage decrease in the value of the capacitance as the capacitor degrades over the aging time. Both parameters change through the aging experiment and are good candidates to be considered as precursor to failure features for the prognostics algorithm. As per standards MILC-62F (2008), a capacitor is considered unhealthy, if under electrical operation, its ESR increases by $280-300 \%$ of its initial value; or the capacitance decreases by $20 \%$ below its pristine condition value. This information is used to set a crisp failure threshold for the RUL estimation process.

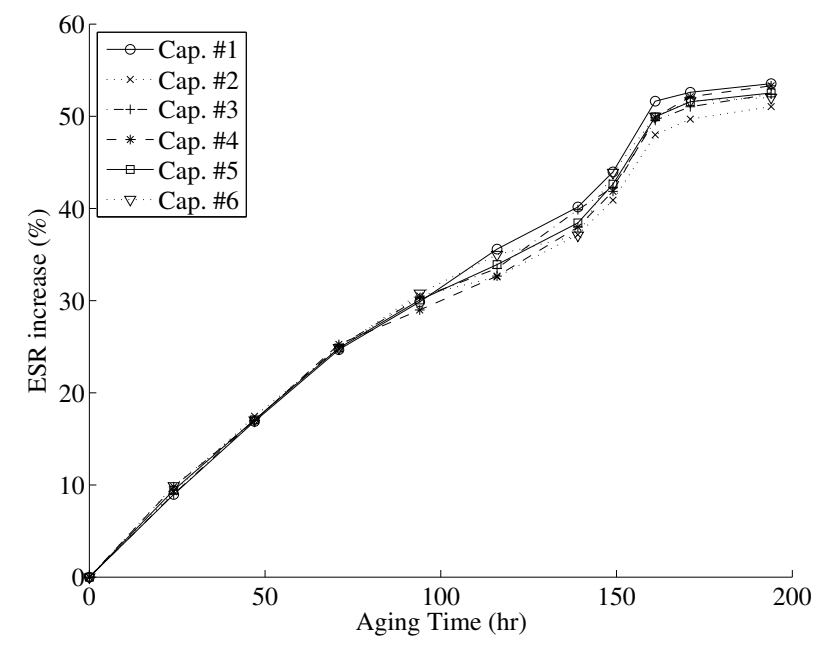

Figure 7. Percentage ESR increase as a function of time.

From the plots in Figure 7 we observe that for total experi- ment time, the ESR value increased by $54 \%-55 \%$ for all the capacitors; while over the same period of time, the capacitance decreased by more than $20 \%$ (the threshold mark for a healthy capacitor) (see Figure 8). As a result, the percentage capacitance loss is selected as a precursor to failure variable to be used in the degradation model development presented next.

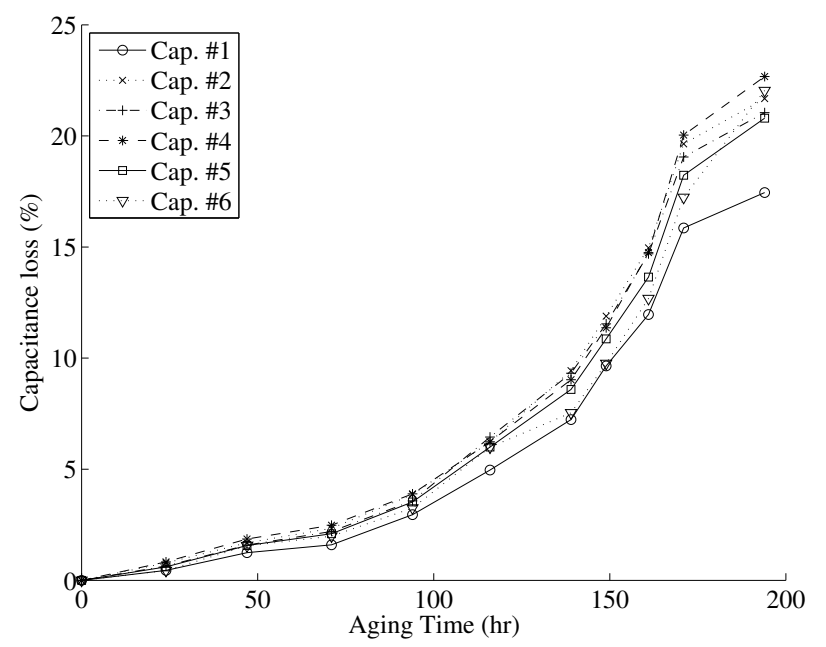

Figure 8. Percentage capacitance loss as a function of time.

\section{Degradation Modeling for Prognostics}

This section presents the details of the degradation model development. A degradation model is an essential part of a model-based prognostics algorithm and it is typically application dependent. A model is formulated based on the empirical evidence of the time evolution of the degradation process from experiments presented in the previous section, particularly, capacitance loss as illustrated by Figure 8 .

\subsection{Nominal operation model}

The non-ideal capacitor model $\mathcal{M}_{1}$ can be used as part of electronics circuits that make use of capacitors. An example is the low-pass filter implementation in Figure 9. In this circuit, input voltage $V_{i}$ is considered as the voltage to be filtered and the voltage across the capacitor (this includes $R_{E}$ as well) is the output voltage $V_{o}$ that is filtered. Let $v(t)=V_{o}(t)$ and $u(t)=V_{i}(t)$ in the low-pass system circuit with non-ideal capacitor shown in Figure 9. A state-space realization model $\left(\mathcal{M}_{2}\right)$ of the dynamic system is given by

$$
\mathcal{M}_{2}:\left\{\begin{aligned}
\dot{z}(t) & =\frac{-1}{C_{R}\left(R+R_{E}\right)} z+\frac{1}{C_{R}\left(R+R_{E}\right)} u(t), \\
v(t) & =\left[1-\frac{R_{E}}{R+R_{E}}\right] z+\frac{R_{E}}{R+R_{E}},
\end{aligned}\right.
$$

where $z(t)=V_{C}(t)$ is the state variable representing the capacitor voltage, $C_{R}, R_{E}$ and $R$ are system parameters. 
Furthermore, $C_{R}$ and $R_{E}$ are parameters that will change through time as the capacitor degrades, but are considered as static (constant) for the nominal operation model $\mathcal{M}_{2}$. Model $\mathcal{M}_{2}$ describes the nominal dynamics of a low-pass filter with a non-ideal capacitor. This model by itself is not sufficient to implement a model-based prognostics algorithm since the degradation process as reflected on model parameters is not modeled. Degradation models describing the time evolution of $R_{E}(t)$ or $C_{R}(t)$ are needed in order to enhance $\mathcal{M}_{1}$ or $\mathcal{M}_{2}$ for model-based prognostics. Nevertheless, $\mathcal{M}_{1}$ or $\mathcal{M}_{2}$ are useful in this form for model-based fault detection and isolation, which is not covered in this work.

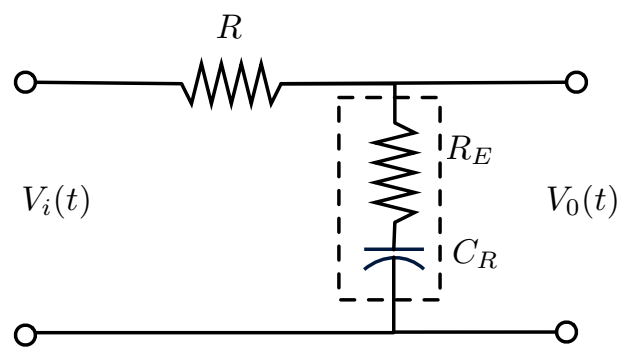

Figure 9. Circuit schematic for a low pass filter with nonideal capacitor $\left(\operatorname{model} \mathcal{M}_{2}\right.$ ).

\subsection{Degradation model}

The percentage loss in capacitance is used as a precursor of failure variable and it is used to build a model of the degradation process. This model relates aging time to the percentage loss in capacitance. Let $C_{l}(t)$ be the percentage loss of capacitance due to degradation as shown by Figure 8 . The following equation represents a dynamic first-order degradation model $\left(\mathcal{D}_{1}\right)$ of the capacitance parameter in the non-ideal capacitor model $\mathcal{M}_{1}$.

$$
\mathcal{D}_{1}: C_{l}(t)=e^{\alpha t}+\beta,
$$

Here, $\alpha$ and $\beta$ are degradation model parameters that will be estimated from the experimental data of accelerated aging experiments (degradation trajectories from Figure 8).

In order to estimate the model parameters, five capacitors are used for estimation, and the remaining capacitor is used to test the prognostics algorithm. This results in five leave-oneout cases for validation of the prognostics algorithm results. A nonlinear least-squares regression algorithm is used to estimate the model parameters $\alpha$ and $\beta$. Table 1 presents the definition of the test cases and the parameter estimation results. The parameter estimates $(\tilde{\alpha}, \tilde{\beta})$ and corresponding $95 \%$ confidence intervals are presented for parameters $\alpha$ and $\beta$. In addition, the error variance $\left(\sigma_{v}^{2}\right)$ is included as a way to assess the quality of the estimation.

Figure 10 shows the estimation results for test case $T_{6}$. The experimental data are presented together with results from the exponential fit function. It can be observed from the residuals that the estimation error increases with time. This is to be expected since the last data point measured for all the capacitors fall slightly off the exponential model. It should be noted that this degradation model with static parameters would be used in a Bayesian tracking framework as an online recursive estimation of $C_{l}(t)$. This will help to overcome the degradation model limitation to represent the behavior close to the failure threshold given the tracking framework ability to compensate the estimation as measurements become available.
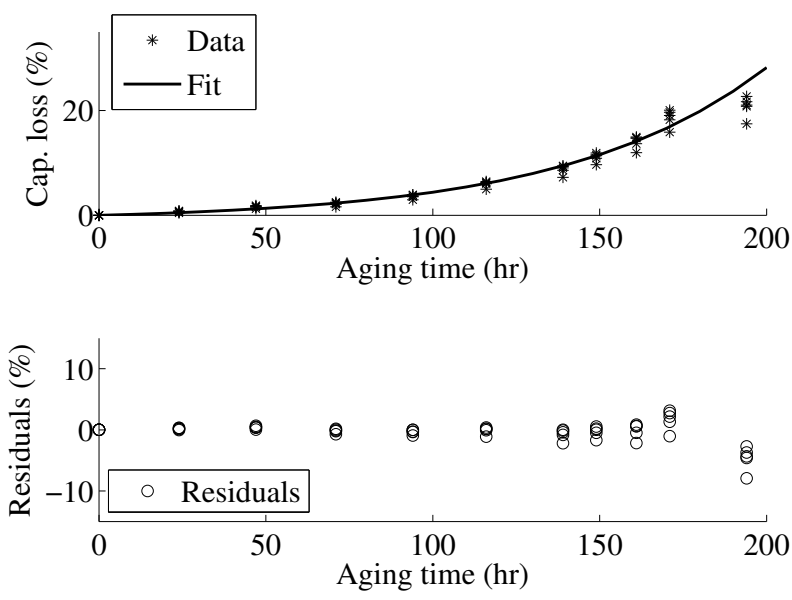

Figure 10. Estimation results for the empirical degradation model.

\subsection{State-space realization for tracking}

The estimated degradation model is used as part of a Bayesian tracking framework to be implemented using the Kalman filter technique. This method requires a state-space dynamic model relating the degradation level at time $t_{k}$ to the degradation level at time $t_{k-1}$. The procedure to obtain a state-space model for $\mathcal{D}_{1}$ is as follows. The non-linear (in time evolution) exponential behavior described in the model is represented as a first order differential equation, which can represent the time evolution of $C_{l}(t)$. Then, the model is discretized in time in order to obtain a discrete-time state-space model $\mathcal{D}_{2}$. From Eq. (3) we have that $C_{l}(t)=e^{\alpha t}+\beta$, taking the first derivative with respect to time and substituting $e^{\alpha t}=C_{l}(t)-\beta$ from Eq. (3) we have

$$
\dot{C}_{l}=\frac{d C_{l}(t)}{d t}=\alpha C_{l}(t)-\alpha \beta .
$$

Taking the finite difference approximation for $\dot{C}_{l}$ with time interval $\Delta t$ we have

$$
\begin{gathered}
\frac{C_{l}(t)-C_{l}(t-\Delta t)}{\Delta t}=\alpha C_{l}(t-\Delta t)-\alpha \beta, \text { and } \\
C_{l}(t)=\left(1+\alpha \Delta_{t}\right) C_{l}(t-\Delta t)-\alpha \beta \Delta_{t} .
\end{gathered}
$$

Letting $t_{k}=t$ and $t_{k-1}=t-\Delta t$ we get the state-space degradation model

$$
\mathcal{D}_{2}: C_{l}\left(t_{k}\right)=\left(1+\alpha \Delta_{k}\right) C_{l}\left(t_{k-1}\right)-\alpha \beta \Delta_{k} .
$$




\begin{tabular}{|c|c|c|c|c|c|}
\hline $\begin{array}{c}\text { Validation } \\
\text { test }\end{array}$ & $\begin{array}{c}\text { Test } \\
\text { capacitor }\end{array}$ & $\begin{array}{c}\text { Training } \\
\text { capacitors }\end{array}$ & $\begin{array}{c}\tilde{\alpha} \\
(95 \% \mathrm{CI})\end{array}$ & $\begin{array}{c}\tilde{\beta} \\
(95 \% \mathrm{CI})\end{array}$ & $\sigma_{v}^{2}$ \\
\hline \hline$T_{2}$ & $\# 2$ & $\# 1, \# 3-\# 6$ & $\begin{array}{c}\mathbf{0 . 0 1 6 2} \\
(0.0160,0.0164)\end{array}$ & $\begin{array}{c}\mathbf{- 0 . 8 3 9 8} \\
(-1.1373,-0.5423)\end{array}$ & 1.8778 \\
\hline$T_{3}$ & $\# 3$ & $\# 1, \# 2, \# 4-\# 6$ & $\begin{array}{c}\mathbf{0 . 0 1 6 2} \\
(0.0160,0.0164)\end{array}$ & $\begin{array}{c}\mathbf{- 0 . 8 2 8 7} \\
(-1.1211,-0.5363)\end{array}$ & 1.9654 \\
\hline$T_{4}$ & $\# 4$ & $\# 1-\# 3, \# 5, \# 6$ & $\begin{array}{c}\mathbf{0 . 0 1 6 1} \\
(0.0159,0.0162)\end{array}$ & $\mathbf{- 0 . 8 2 1 7}$ & \\
\hline$T_{5}$ & $\# 5$ & $\# 1.1125,-0.5308)$ & 1.8860 \\
\hline$T_{6}$ & $\# 6$ & $\# 1-\# 6$ & $\begin{array}{c}\mathbf{0 . 0 1 6 2} \\
(0.0161,0.0164)\end{array}$ & $(-1.1134,-0.4560)$ & 2.1041 \\
\hline
\end{tabular}

Table 1. Degradation model $\left(\mathcal{D}_{1}\right)$ parameter estimation results.

This model can be used in a Bayesian tracking framework in order to continuously estimate the value of the loss in capacitance through time as measurement become available.

\section{Model-based Prognostics Framework}

A model-based prognostics algorithm based on Kalman filter and a physics inspired empirical degradation model is presented. The methodology consists of the following three main steps and it is depicted in Figure 11.

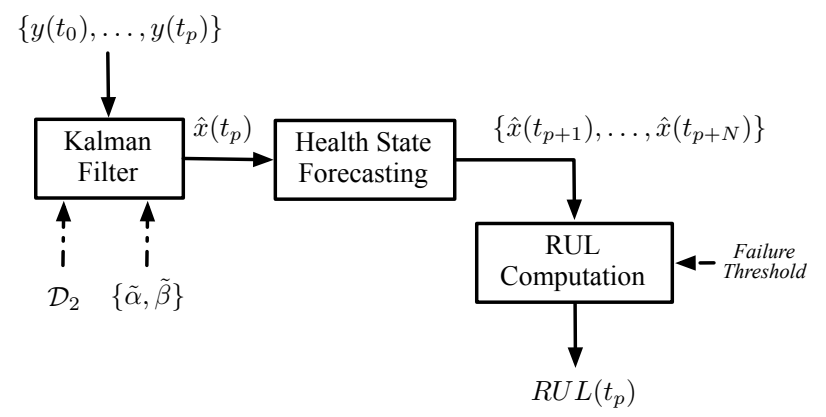

Figure 11. Model-based prognostics methodology

1. State tracking (Kalman Filter): The capacitance loss $C_{l}(t)$ is defined as the state variable to be estimated and the degradation model is expressed as a discrete time dynamic model $\left(\mathcal{D}_{2}\right)$ in order to estimate capacitance loss as new measurements become available. Direct measurements of the capacitance are available for the filtering process.

2. Health state forecasting: It is necessary to forecast the state variable once there are no more measurements available at time of RUL prediction $t_{p}$. This is done by evaluating the degradation model through time using the state estimate at time $t_{p}\left(\hat{x}\left(t_{p}\right)\right)$ as initial value.

3. Remaining life computation: $R U L\left(t_{p}\right)$ is computed as the time difference between time of prediction $t_{p}$ and the end-of-life (EOL) time. End of life is defined as the time at which the forecasted state crosses the failure threshold value.
This process is repeated for different values of $t_{p}$ through the life of the component under consideration.

\subsection{Kalman filter for state estimation}

A state-space dynamic model is needed for the state estimation. The state variable $x_{k}$ at time $t_{k}$ is defined as the percentage capacitance loss $C_{l}(k)$. Since the system measurements are percentage loss in capacitance as well, the output equation is given by $y_{k}=h x_{k}$, where the value of $h$ is equal to one. The following system structure is used in the implementation of the state estimation using the Kalman filter.

$$
\begin{aligned}
& x_{k}=A_{k} x_{k-1}+B_{k} u+v, \\
& y_{k}=h x_{k}+w,
\end{aligned}
$$

where,

$$
\begin{aligned}
A_{k} & =\left(1+\Delta_{k}\right), \\
B_{k} & =-\alpha \beta \Delta_{k}, \\
h & =1, \\
u & =1 .
\end{aligned}
$$

The time increment between measurements $\Delta_{k}$ is not constant since measurements were taken at non-uniform sampling rate. This implies that some of the parameters of the model in Eq. (6) will change through time. Furthermore, $v$ and $w$ are normal random variables with zero mean and $Q$ and $R$ variance respectively. The description of the Kalman filtering algorithm is omitted from this article. A thorough description of the algorithm can be found in Stengel (1994), a description of how the algorithm is used for forecasting can be found in Chatfield (2003) and an example of its usage for prognostics can be found in (Saha et al., 2009).

\subsection{Future state forecasting}

The use of the dynamic degradation model for health-state forecasting requires the time evolution of the state without updating the error covariance matrix and the posterior distribution of the state vector. Basically, the infrastructure pro- 
vided by the Kalman filter for updating the state given new measurements on the system is not required in this step. The state equation from the discrete-time system in Eq. (6) is evaluated recursively $n$ times ensuring the forecasted state $\hat{x}_{p+n}$ crosses the failure threshold. The noise variable $v$ is also omitted. The $n$ step ahead forecasting equation is given below by Eq. (8). The last update is done at the time of the last measurement $t_{l}$. Note that $t_{l}=t_{p}$ for this particular prognostics implementation. This is due to the authors' decision to make RUL predictions at each time a new measurement is available.

$$
\hat{x}_{p+n}=A^{n} x_{p}+\sum_{i=0}^{n-1} A^{i} B
$$

The subscripts from parameters $A$ and $B$ are omitted since a constant $\Delta_{t}$ is used in the forecasting mode (one prediction every hour).

\subsection{Remaining useful life computation}

Computing the RUL based on the forecasting of the health states requires the identification of the EOL time based on the failure threshold. Defining $R U L\left(t_{p}\right)$ as the remaining useful life,

$$
R U L\left(t_{p}\right)=t_{E O L}-t_{p}
$$

The time at end-of-life $\left(t_{E O L}\right)$ is a continuous variable which is computed from the forecast $\hat{x}_{p+n}$. Let $\hat{x}_{p+j}$ be the first forecast value to cross the failure threshold. A linear interpolation between $\hat{x}_{p+j}$ and $\hat{x}_{p+j-1}$ is used to compute $t_{E O L}$.

\subsection{Noise models}

The model noise variance $Q$ was estimated from the model regression residuals for each test case presented in Table 1. The variance is listed in the last column Table 1. This variance was used for the model noise in the Kalman filter implementation. The measurement noise variance $R$ is also required in the filter implementation. This variance was computed from the direct measurements of the capacitance with the EIS equipment, the observed variance is $4.99 \times 10^{-7}$.

\section{Prediction of Remaining USEFul Life Results}

State estimation and RUL prediction results are discussed for test case $T_{6}$. Figure 12 shows the result of the filter tracking the degradation signal. The residuals show an increased error with aging time. This is to be expected given the results observed from the model estimation process. It should be noted that the scale of the magnitude of the residuals is $10^{-7}$. This is to be expected given the capability of the Bayesian tracking methods to make corrections to the state estimates based on direct or indirect measurements of the state variable being estimated. Even though the empirical degradation model with static parameters $\mathcal{D}_{1}$ does not perfectly represent the observed degradation data (Figure 10), the Kalman filter is able to make the appropriate corrections resulting on a good online state estimation performance.
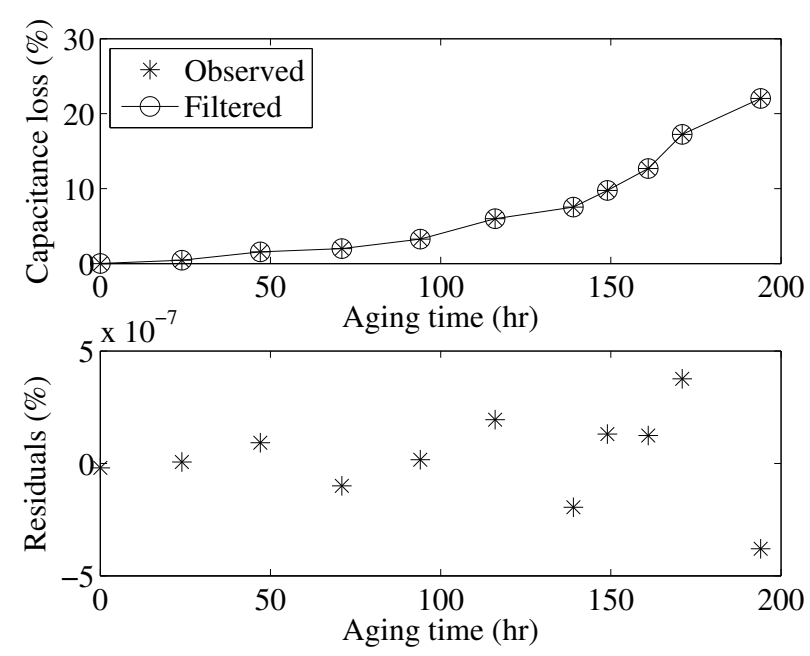

Figure 12. Tracking results for the Kalman filter implementation applied to test case $T_{6}$.

Figure 13 presents results from the remaining useful life prediction algorithm at time $t_{p}=161(\mathrm{hr})$, which is the time at which $E S R$ and $C$ measurements are taken. The failure threshold is considered to be a crisp value of $20 \%$ decrease in capacitance. End of life is defined as the time at which the forecasted percentage capacity loss trajectory crosses the failure threshold. Therefore, RUL is $t_{E O L}$ minus 161 hours. Figures 14 and 15 present the tracking and forecasting results for test case $T_{6}$. Appendix A presents the prediction plots for the remaining of the validation cases.

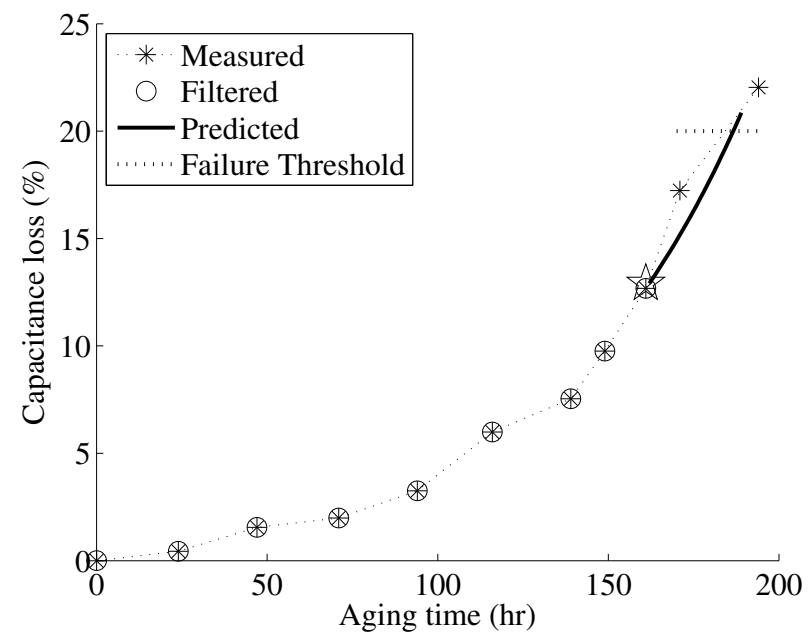

Figure 13. RUL prediction at time $161(\mathrm{hr})$ for test case $T_{6}$. 


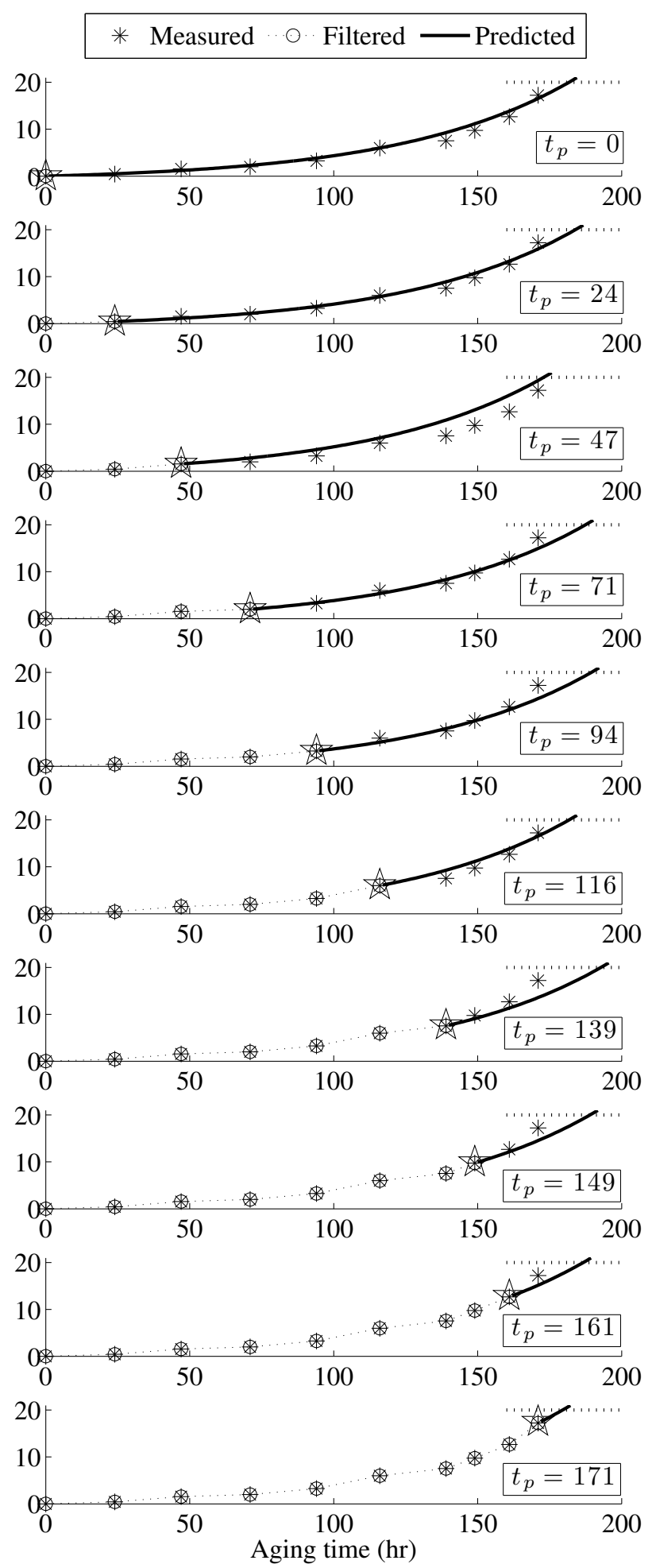

Figure 14. $T_{6}$ : Health state estimation and forecasting of capacitance loss $(\%)$ at different times $t_{p}$ during the aging time; $t_{p}=[0,24,47,71,94,116,139,149,161,171]$.

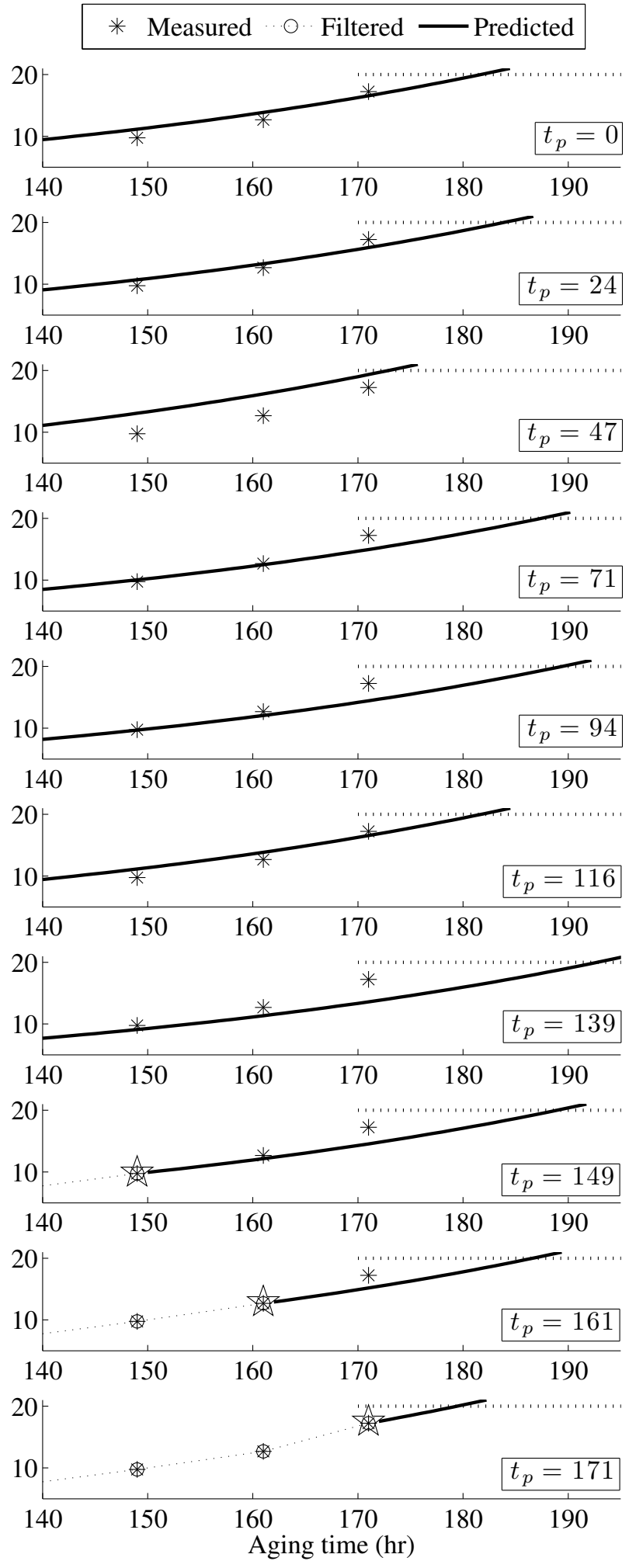

Figure 15. $T_{6}$ : Detail of the health state estimation and forecasting of capacitance loss (\%) at different times $t_{p}$ during the aging time; $t_{p}=[0,24,47,71,94,116,139,149,161,171]$. 
Figure 14 illustrates the health-state estimation process and forecasting for the capacitance loss at different points during the aging time $\left(t_{p}=\right.$ $[0,24,47,71,94,116,139,149,161,171]$ hours). The star symbol depicts the final state update (Kalman filter output) and the bold continuous line represents the state forecasted until the failure threshold is crossed. The failure threshold is depicted with a dotted horizontal line at $20 \%$. RUL estimations are made after each point in which measurements are available. It can be observed that the predictions become better as the prediction is made closer to the actual EOL. This is possible because the estimation process has more information to update the estimates as it nears EOL. Figure 15 presents a zoomed-in version of Figure 14 focusing in the area close to the failure threshold.

An $\alpha-\lambda$ prognostics performance metric is presented in Figure 16 for validation test $T_{6}$. The continuous black line $\left(R U L^{*}\right)$ represents ground truth and the shaded region corresponds to a $30 \%(\alpha=0.3)$ error bound in the RUL prediction. This metric specifies that the prediction be within the error bound halfway between the first prediction and EOL $(\lambda=0.5)$. In addition, this metric allows us to visualize how the RUL prediction performance changes as data closer to EOL becomes available. Appendix B presents the $\alpha-\lambda$ metric plots for the remaining validation cases. Details on the prognostics performance metrics used in this work are available in Saxena, Celaya, Saha, Saha, and Goebel (2010).

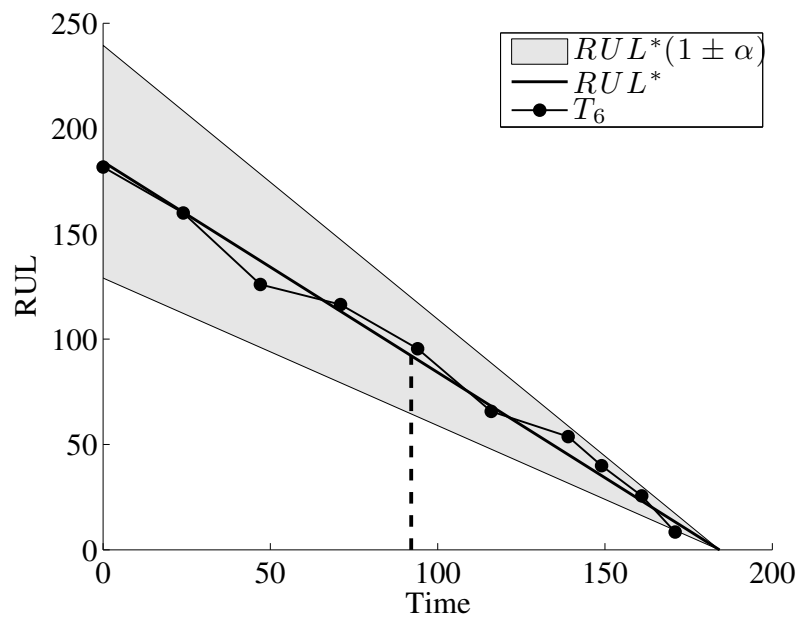

Figure 16. Performance based on $\alpha-\lambda$ performance metric.

\subsection{Validation tests}

Since the total number of capacitors used for the experiment was small (six), a leave-one out validation approach was used to assess the validity of the methodology. In this approach, the prognostics algorithm was executed in a single capacitor out of the set of six available. The remaining five capacitors are used for the parameter estimation of the degradation model. Only five of the six available capacitors crossed the $20 \%$ capacitance loss failure threshold, as a result, only five validation cases were considered.

Table 2 shows performance based on the relative accuracy (RA) metric in Eq. (10). The RA metric allows for an assessment of the percentage accuracy relative to the ground-truth value. RA values of 100 represent perfect accuracy. The RA is presented for all the test cases for different prediction times. The last column of Table 2 represents the median RA of all the test cases for a particular prediction time. It is observed that the RA values decrease considerably for $t_{p}=171$. This is consistent with previous observations indicating that the algorithm with a fixed-parameter model is not able to cope with the sudden jump in exponential behavior present around the 171 hour. This is a limitation that could be overcome by either an enhanced degradation model or a an online estimation of degradation model parameters using a more sophisticated Bayesian tracking method like extended Kalman filter or particle filter. It should be noted that the tracking algorithm has only ten measurement available to update the estimates, this has also an impact on the algorithm's ability to converge quickly to a small state estimation error. Having more measurements available through the aging experiments will have a positive impact on the prognostics performance.

$$
R A=100\left(1-\frac{\left|R U L^{*}-R U L^{\prime}\right|}{R U L^{*}}\right)
$$

\begin{tabular}{|c|c|c|c|c|c|c|}
\hline$t_{p}$ & $R A_{T 2}$ & $R A_{T 3}$ & $R A_{T 4}$ & $R A_{T 5}$ & $R A_{T 6}$ & $\widetilde{R A}$ \\
\hline \hline 24 & 94.8 & 95.5 & 91.9 & 96.9 & 99.7 & $\mathbf{9 5 . 5}$ \\
\hline 47 & 97.4 & 99.3 & 96.4 & 96.7 & 91.7 & $\mathbf{9 6 . 7}$ \\
\hline 71 & 87.5 & 91.9 & 84.5 & 94.1 & 97.1 & $\mathbf{9 1 . 9}$ \\
\hline 94 & 85.6 & 90 & 78.9 & 94.8 & 94.2 & $\mathbf{9 0}$ \\
\hline 116 & 86 & 99.1 & 76.5 & 98 & 96.2 & $\mathbf{9 6 . 2}$ \\
\hline 139 & 77.8 & 95.8 & 53.1 & 96.7 & 81.1 & $\mathbf{8 1 . 1}$ \\
\hline 149 & 82.1 & 98.4 & 46.9 & 94.8 & 86.6 & $\mathbf{8 6 . 6}$ \\
\hline 161 & 77.2 & 87.3 & 16.6 & 87.5 & 89.8 & $\mathbf{8 7 . 3}$ \\
\hline 171 & 26.6 & 26.4 & N/A & 34.8 & 63.7 & $\mathbf{3 0 . 7}$ \\
\hline \hline
\end{tabular}

Table 2. Validation based on relative accuracy (RA) metric.

Table 3 summarizes results for the remaining life prediction $\left(R U L^{\prime}\right)$ for all the test cases. The predictions at all points in time where measurements are available are included. The second column $\left(R U L^{*}\right)$ indicates the RUL ground-truth use for computation of prediction errors. The results of the methodology are presented visually with the tracking and forecasting plots, the $\alpha-\lambda$ metric. The tabular results are included to provide needed information to compute other prognostics performance metrics and for algorithm performance comparison under the same run to failure dataset. 


\begin{tabular}{|c|c|c|c|c|c|c|}
\hline$t_{p}$ & $R U L^{*}$ & $R U L_{T 2}^{\prime}$ & $R U L_{T 3}^{\prime}$ & $R U L_{T 4}^{\prime}$ & $R U L_{T 5}^{\prime}$ & $R U L_{T 6}^{\prime}$ \\
\hline \hline 24 & 151.04 & 158.84 & 164.88 & 158.76 & 167.76 & 159.89 \\
47 & 128.04 & 131.32 & 134.08 & 128.35 & 135.32 & 125.91 \\
71 & 104.04 & 117.01 & 119.88 & 115.37 & 122.63 & 116.41 \\
94 & 81.04 & 92.69 & 96.64 & 93.09 & 97.6 & 95.42 \\
116 & 59.04 & 67.28 & 65.39 & 67.77 & 69.5 & 65.71 \\
139 & 36.04 & 44.01 & 44.72 & 46.88 & 49.4 & 53.75 \\
149 & 26.04 & 30.67 & 32.41 & 33.55 & 35.92 & 39.95 \\
161 & 14.04 & 17.23 & 18.28 & 18.2 & 22.64 & 25.6 \\
171 & 4.04 & 1.07 & 2.89 & N/A & 5.52 & 8.45 \\
\hline
\end{tabular}

Table 3. Summary of RUL forecasting results for all test cases.

\section{CONClusion}

This paper presents a RUL prediction algorithm based on accelerated life test data and an empirical degradation model. The main contributions of this work are:

1. the identification of the lumped-parameter model (Figure 5) for a real capacitor as a viable reduced-order model for prognostics-algorithm development;

2. the identification of the ESR and C model parameters as precursor of failure features;

3. the development of an empirical degradation model based on accelerated life test data which accounts for shifts in capacitance as a function of time;

4. the implementation of a Bayesian based health state tracking and remaining useful life prediction algorithm based on the Kalman filtering framework.

One major contribution of this work is the prediction of remaining useful life for capacitors as new measurements become available. This capability increases the technology readiness level of prognostics applied to electrolytic capacitors.

\subsection{Ongoing and future work}

The results presented here are based on accelerated life test data and on the accelerated life timescale. Further research will focus on development of functional mappings that will translate the accelerated life timescale into real usage conditions time-scale, where the degradation process dynamics will be slower, and subject to several types of stresses.

The performance of the proposed exponential-based degradation model is satisfactory for this study based on the quality of the model fit to the experimental data and the RUL prediction performance as compared to ground truth. As part of future work we will also focus on the exploration of additional models based on the physics of the degradation process and larger sample size for aged devices.

Additional experiments are currently underway to increase the number of test samples. This will greatly enhance the quality of the model, and guide the exploration of additional degradation-models, where the loading conditions and the environmental conditions are also accounted for towards degradation dynamics.

\section{ACKNOWLedgment}

This work was funded by the NASA Aviation Safety Program, SSAT project.

\section{NOMENClature}

$C_{I} \quad$ Ideal capacitance value for an ideal capacitor

$C_{R} \quad$ Real capacitor value for a non-ideal capacitor model

$R_{E} \quad$ Equivalent series resistance of the capacitor

$C_{l}(k) \quad$ Capacitance percentage loss at time $t_{k}$ (state variable)

$T_{i} \quad$ Validation test on capacitor $i$

$\mathcal{M}_{i} \quad$ Nominal model for a component or system

$\mathcal{D}_{i} \quad$ Degradation model for a capacitor

$R_{L} \quad$ Load resistance on electrical overstress system

$V_{L} \quad$ Load voltage on electrical overstress system

$V_{o} \quad$ Electrical overstress voltage in aging system

$Z_{I} \quad$ Ideal capacitor impedance

$Z \quad$ Capacitor impedance for non-ideal capacitor model $\mathcal{M}_{1}$

\section{REFERENCES}

Bhatti, U., \& Ochieng, W. (2007). Failure modes and models for integrated GPS/INS systems. The Journal of Navigation, 60, 327.

Celaya, J., Kulkarni, C., Biswas, G., \& Goebel, K. (2011a, September). A Model-based Prognostics Methodology for Electrolytic Capacitors Based on Electrical Overstress Accelerated Aging. In Proceedings of the Annual Conference of the Prognostics and Health Management Society. Montreal, Canada.

Celaya, J., Kulkarni, C., Biswas, G., \& Goebel, K. (2011b, March). Towards Prognostics of Electrolytic Capacitors. In In Proceedings of the AIAA Infotech@Aerospace Conference. St. Louis, MO.

Celaya, J., Kulkarni, C., Biswas, G., \& Goebel, K. (2012, 
January). Prognostic and Experimental Techniques for Electrolytic Capacitor Health Monitoring. In Proceedings of the The Annual Reliability and Maintainability Symposium (RAMS). Reno, Nevada.

Chatfield, C. (2003). The Analysis of Time Series: An Introduction (6th ed.). Chapman and Hall/CRC.

Goodman, D., Hofmeister, J., \& Judkins, J. (2007). Electronic prognostics for switched mode power supplies. Microelectronics Reliability, 47(12), 1902-1906.

Gu, J., Azarian, M. H., \& Pecht, M. G. (2008). Failure prognostics of multilayer ceramic capacitors in temperature-humidity-bias conditions. In International Conference on Prognostics and Health Management (p. 1-7). Denver, CO.

International Standard IEC 60384-4-1: Fixed capacitors for use in electronic equipment (3rd ed.). (2007). International Electrotechnical Commission (IEC).

Judkins, J. B., Hofmeister, J., \& Vohnout, S. (2007). A Prognostic Sensor for Voltage Regulated Switch-Mode Power Supplies. In IEEE Aerospace Conference (p. 1$8)$.

Kulkarni, C., Biswas, G., Bharadwaj, R., \& Kim, K. (2010). Effects of Degradation in DC-DC Converters on Avionics Systems: A Model Based Approach. In Machinery Failure Prevention Technology Conference, MFPT.

Kulkarni, C., Biswas, G., \& Koutsoukos, X. (2009). A prognosis case study for electrolytic capacitor degradation in DC-DC converters. In Proceedings of the Annual Conference of the Prognostics and Health Management Soceity. San Diego, CA.

Kulkarni, C., Biswas, G., Koutsoukos, X., Celaya, J., \& Goebel, K. (2010). Integrated diagnostic/prognostic experimental setup for capacitor degradation and health monitoring. In IEEE AUTOTESTCON.

Kulkarni, C., Biswas, G., Koutsoukos, X., Goebel, K., \& Celaya, J. (2010a). Experimental Studies of Ageing in Electrolytic Capacitors. In Proceedings of the Annual Conference of the Prognostics and Health Management Soceity.

Kulkarni, C., Biswas, G., Koutsoukos, X., Goebel, K., \& Celaya, J. (2010b). Physics of Failure Models for Capacitor Degradation in DC-DC Converters. In The Maintenance and Reliability Conference, MARCON.

Kulkarni, C., Celaya, J., Goebel, K., \& Biswas, G. (2012a, September). Bayesian Framework Approach for Prognostic Studies in Electrolytic Capacitor under Thermal Overstress Conditions. In Proceedings of Annual Conference of the Prognostics and Health Management Society. Minneapolis, MN.

Kulkarni, C., Celaya, J., Goebel, K., \& Biswas, G. (2012b). Physics Based Electrolytic Capacitor Degradation Models for Prognostic Studies under Thermal Overstress. In Proceedings of First European Conference of the Prognostics and Health Management Soci- ety. Dresden, Germany.

Kulkarni, C., Celaya, J., Goebel, K., \& Biswas, G. (2012c, June). Prognostics Health Management and Physics based failure Models for Electrolytic Capacitors. In Proceedings of American Institute of Aeronautics and Astronautics, AIAA Infotech@Aerospace Conference. Garden Grove, CA.

MIL-C-62F. (2008). General specification for capacitors, fixed, electrolytic (dc. aluminum, dry electrolyte, polarized),. Military Specification. Department of Defense.

Nie, L., Azarian, M. H., Keimasi, M., \& Pecht, M. (2007). Prognostics of ceramic capacitor temperaturehumidity-bias reliability using Mahalanobis distance analysis. Circuit World, 33(3), 21 - 28.

Orsagh, R., Brown, D., Roemer, M., Dabnev, T., \& Hess, A. (2005). Prognostic health management for avionics system power supplies. In IEEE Aerospace Conference (p. 3585-3591).

Saha, B., Goebel, K., \& Christophersen, J. (2009). Comparison of Prognostic Algorithms for Estimating Remaining Useful Life of Batteries. IEEE Transactions of the Institute of Measurement and Control, 31(3-4), 293-308.

Saxena, A., Celaya, J., Saha, B., Saha, S., \& Goebel, K. (2010). Metrics for offline evaluation of prognostic performance. International Journal of Prognostics and Health Management, 1-1(1).

Stengel, R. F. (1994). Optimal Control and Estimation. Dover Books on Advanced Mathematics.

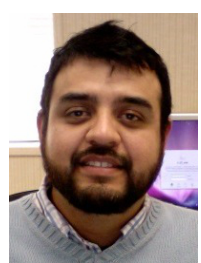

Dr. José R. Celaya is a research scientist with SGT Inc. at the Prognostics Center of Excellence, NASA Ames Research Center. $\mathrm{He}$ received a Ph.D. degree in Decision Sciences and Engineering Systems in 2008, a M. E. degree in Operations Research and Statistics in 2008, a M. S. degree in Electrical Engineering in 2003, all from Rensselaer Polytechnic Institute, Troy New York; and a B. S. in Cybernetics Engineering in 2001 from CETYS University, México.

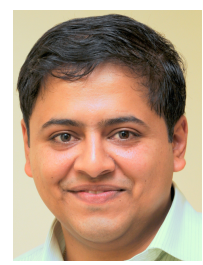

Chetan S. Kulkarni is a Research Assistant at ISIS, Vanderbilt University. He received the M.S. degree in EECS from Vanderbilt University, Nashville, TN, in 2009, where he is currently a Ph.D. candidate and received a B. E. degree in Electronics and Electrical Engineering from University of

Pune, India in 2002. 


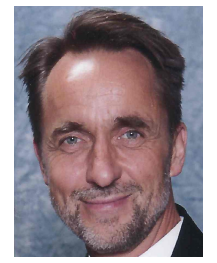

Dr. Kai Goebel is a deputy area lead of the Discovery and Systems Health Technology Area at NASA Ames Research Center. He also coordinates the Prognostics Center of Excellence and is the Technical Lead for Prognostics and Decision Making in NASAs System-wide Safety and Assurance Technologies Project. Prior to joining NASA in 2006, he was a senior research scientist at General Electric Corporate Research and Development center since 1997. Dr. Goebel received his Ph.D. at the University of California at Berkeley in 1996. He has carried out applied research in the areas of real time monitoring, diagnostics, and prognostics and he has fielded numerous applications for aircraft engines, transportation systems, medical systems, and manufacturing systems. He holds 17 patents and has co-authored more than 250 technical papers in the field of IVHM. Dr. Goebel was an adjunct professor of the CS Department at Rensselaer Polytechnic Institute (RPI), Troy, NY, between 1998 and 2005 where he taught classes in Soft Computing and Applied Intelligent Reasoning Systems. He has been the co-advisor of 6 Ph.D. students. Dr. Goebel is a member of several professional societies, including ASME, AAAI, AIAA, IEEE, VDI, SAE, and ISO. He was the General Chair of the Annual Conference of the PHM Society, 2009, has given numerous invited and keynote talks and held many chair positions at the PHM conference and the AAAI Annual meetings series. He is currently member of the board of directors of the PHM Society and associate editor of the International Journal of PHM.

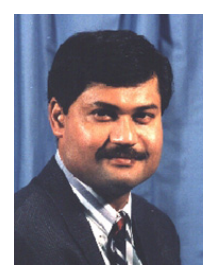

Gautam Biswas is a Professor of Computer Science, Computer Engineering, and Engineering Management in the EECS Department and a Senior Research Scientist at the Institute for Software Integrated Systems (ISIS) at Vanderbilt University. He has an undergraduate degree in Electrical Engineering from the Indian Institute of Technology (IIT) in Mumbai, India, and M.S. and Ph.D. degrees in Computer Science from Michigan State University in E. Lansing, MI.

Prof. Biswas conducts research in Intelligent Systems with primary interests in hybrid modeling, simulation, and analysis of complex embedded systems, and their applications to diagnosis, prognosis, and fault-adaptive control. As part of this work, he has worked on fault diagnosis and fault-adaptive control of secondary sodium cooling systems for nuclear reactors, automobile engine coolant systems, fuel transfer and avionics systems for aircraft, Advanced Life Support systems and power distribution systems for NASA. He has also initiated new projects in health management of complex systems, which includes online algorithms for distributed monitoring, diagnosis, and prognosis. More recently, he is working on data mining for diagnosis, and developing methods that combine model-based and data-driven approaches for diagnostic and prognostic reasoning. This work, in conjunction with Honeywell Technical Center and NASA Ames, includes developing sophisticated data mining algorithms for extracting causal relations amongst variables and parameters in a system. In other research projects, he is involved in developing simulation-based environments for learning and instruction. His industrial collaborators include Airbus, Honeywell Technical Center, and Boeing Research and Development. He has published extensively, and has over 300 refereed publications.

Dr. Biswas is an associate editor of the IEEE Transactions on Systems, Man, and Cybernetics, Prognostics and Health Management, and Educational Technology and Society journal. He has served on the Program Committee of a number of conferences, and most recently was Program co-chair for the 18th International Workshop on Principles of Diagnosis and Program Coordination Chair for the 20th International Conference in Computers in Education. He is currently serving on the Executive committee of the Asia Pacific Society for Computers in Education and is the IEEE Computer Society representative to the Transactions on Learning Technologies steering committee. He is also serving as the Secretary/Treasurer for ACM Sigart. He is a senior member of the IEEE Computer Society, ACM, AAAI, and the Sigma Xi Research Society. 


\section{A. Prognostics validation results}
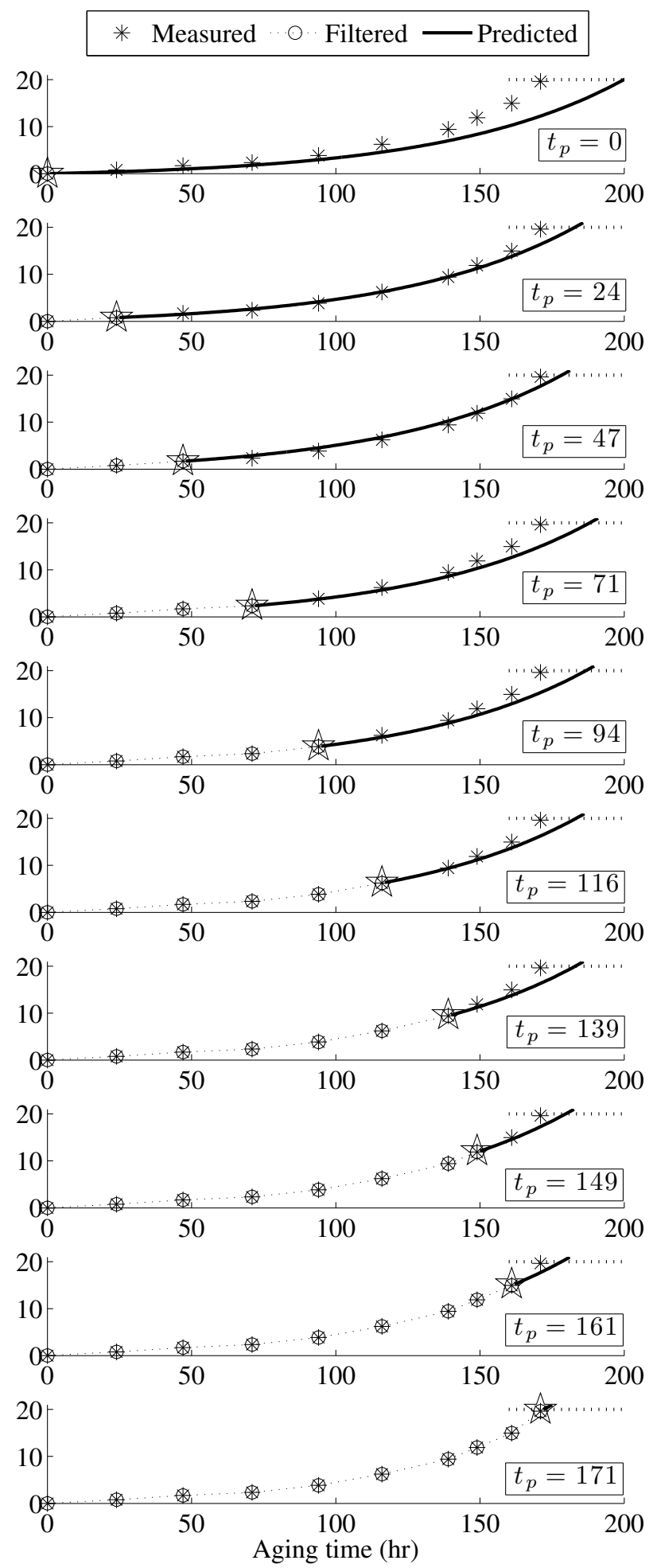

Figure 17. $T_{2}$ : Health state estimation and forecasting of capacitance loss $(\%)$ at different times $t_{p}$ during the aging time; $t_{p}=[0,24,47,71,94,116,139,149,161,171]$.

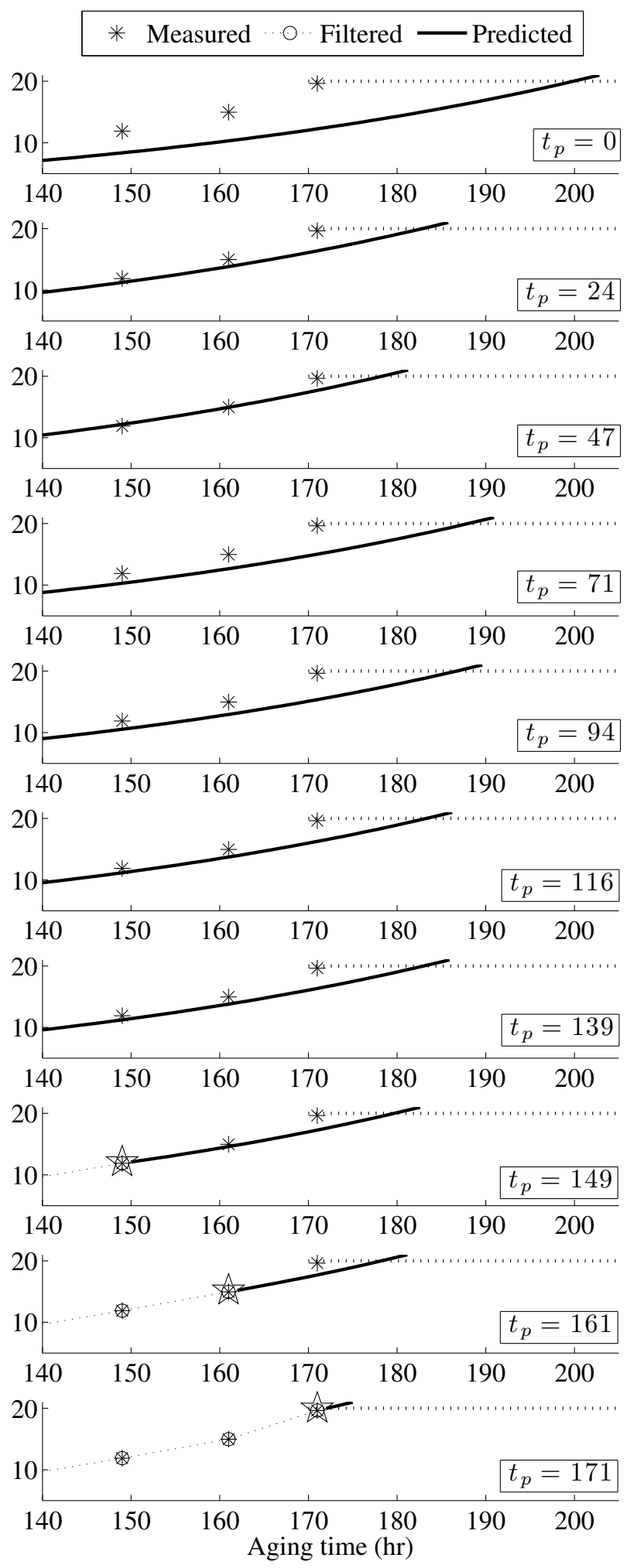

Figure 18. $T_{2}$ : Detail of the health state estimation and forecasting of capacitance loss $(\%)$ at different times $t_{p}$ during the aging time; $t_{p}=[0,24,47,71,94,116,139,149,161,171]$. 


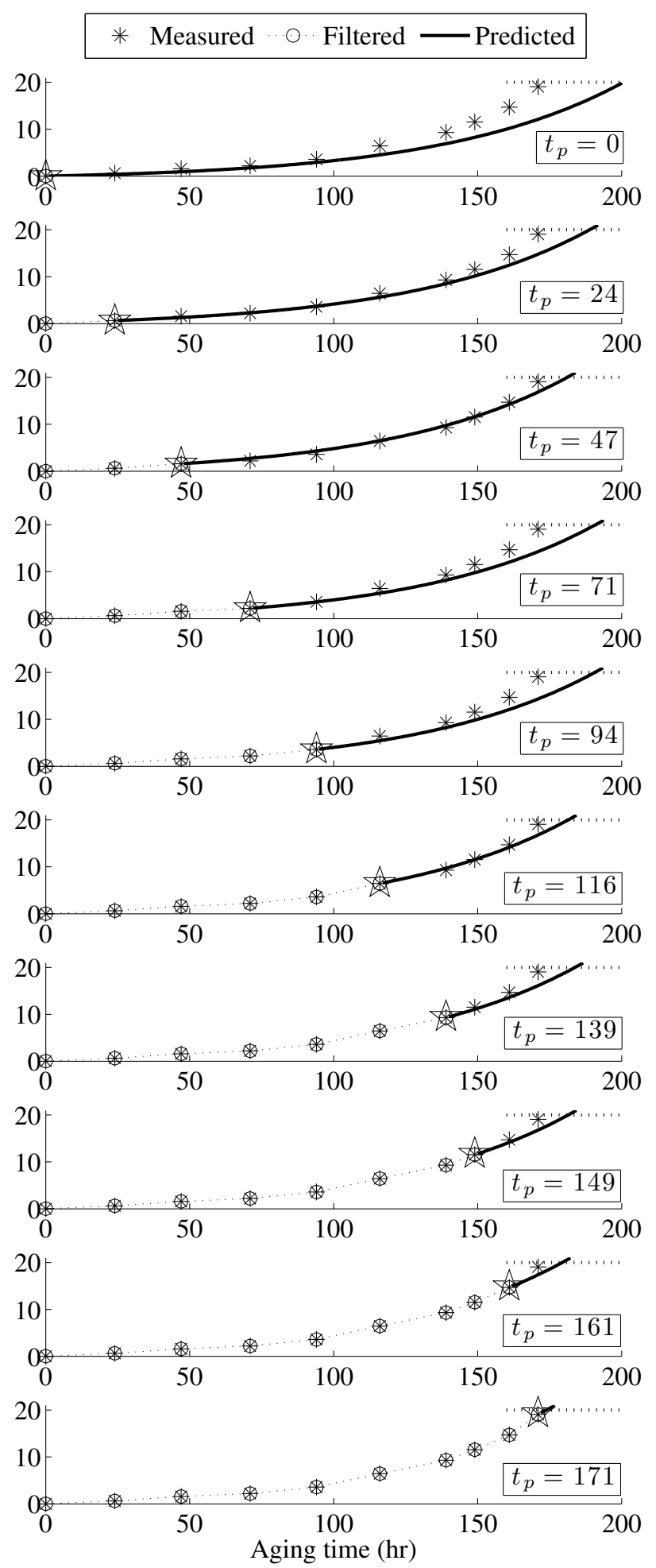

Figure 19. $T_{3}$ : Health state estimation and forecasting of capacitance loss $(\%)$ at different times $t_{p}$ during the aging time; $t_{p}=[0,24,47,71,94,116,139,149,161,171]$.

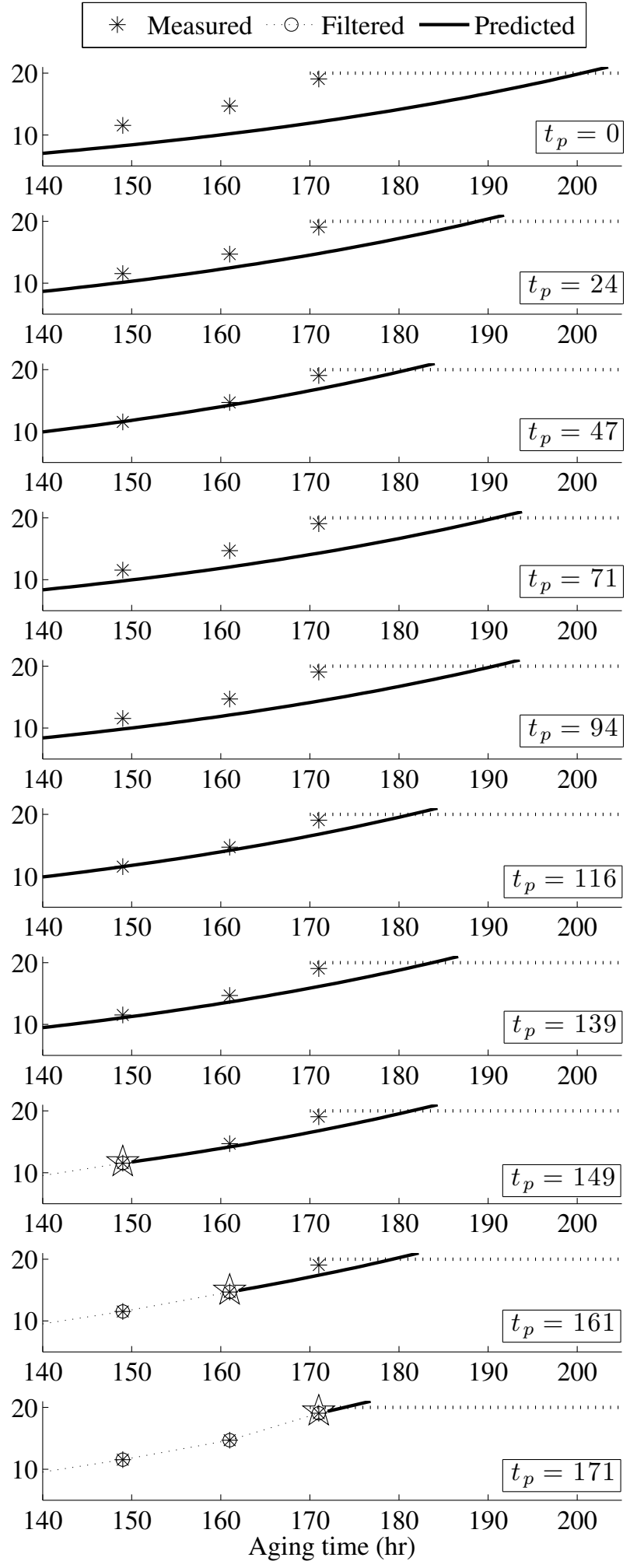

Figure 20. $T_{3}$ : Detail of the health state estimation and forecasting of capacitance loss (\%) at different times $t_{p}$ during the aging time; $t_{p}=[0,24,47,71,94,116,139,149,161,171]$. 


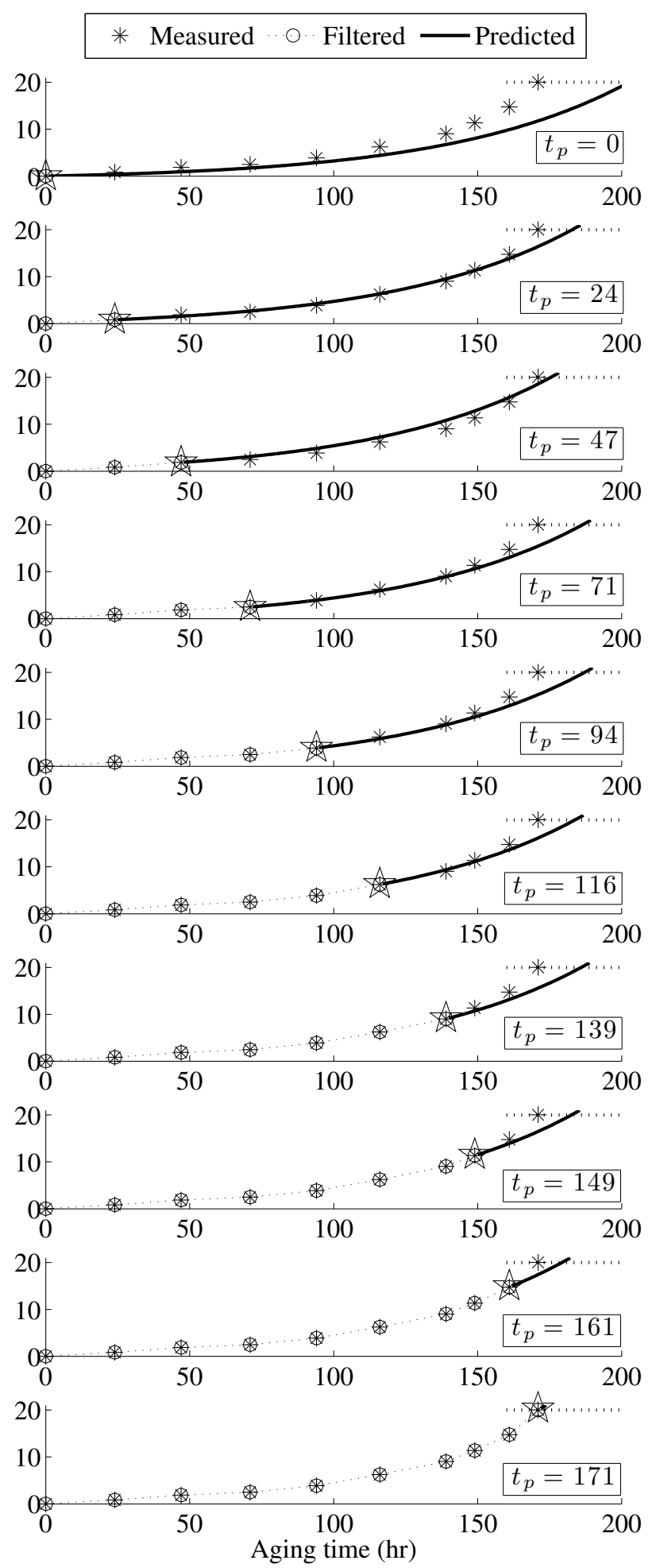

Figure 21. $T_{4}$ : Health state estimation and forecasting of capacitance loss $(\%)$ at different times $t_{p}$ during the aging time; $t_{p}=[0,24,47,71,94,116,139,149,161,171]$.

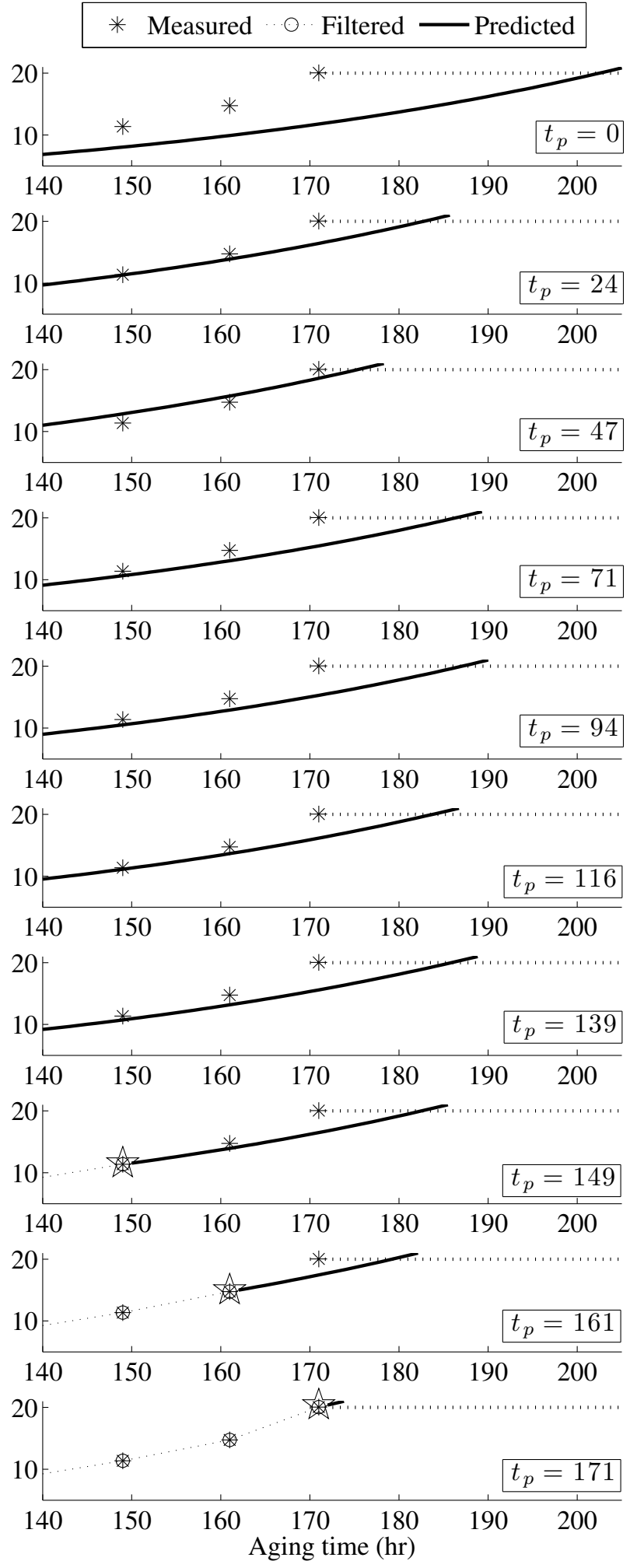

Figure 22. $T_{4}$ : Detail of the health state estimation and forecasting of capacitance loss (\%) at different times $t_{p}$ during the aging time; $t_{p}=[0,24,47,71,94,116,139,149,161,171]$. 


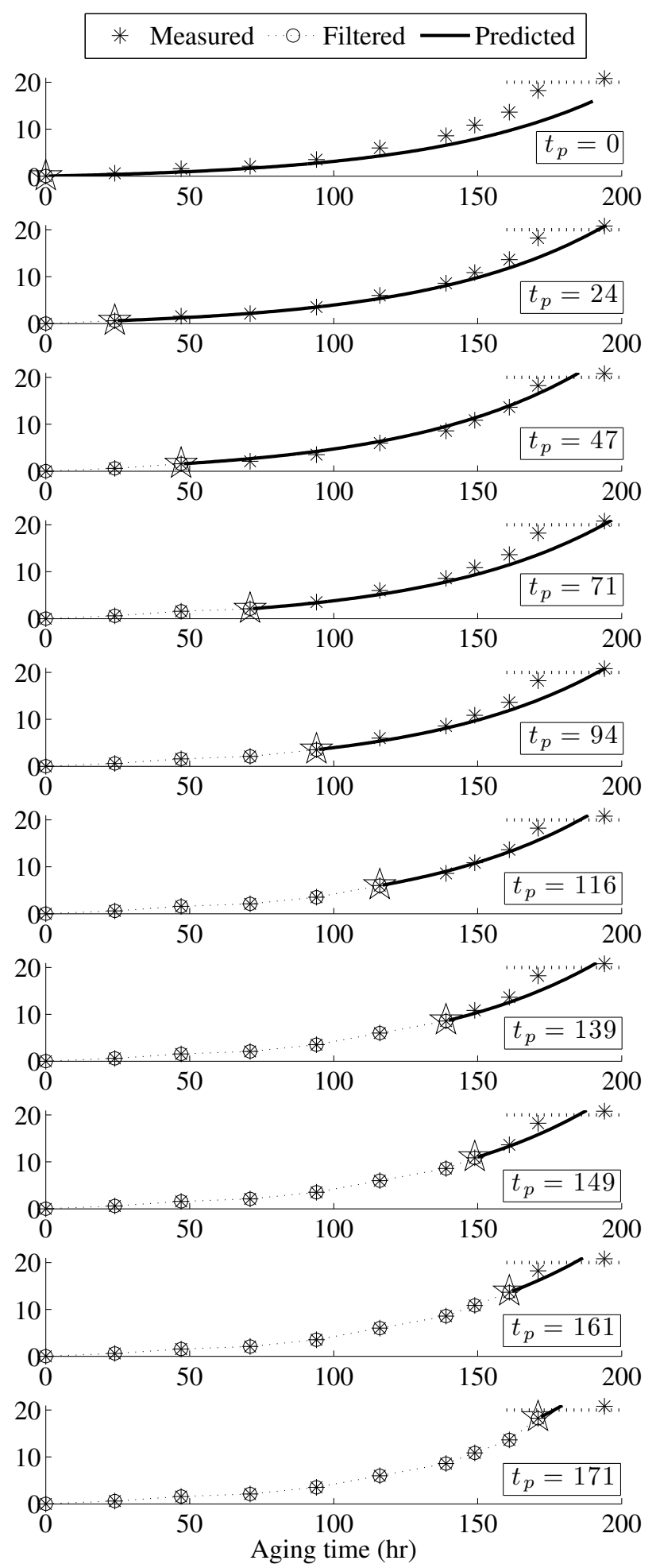

Figure 23. $T_{5}$ : Health state estimation and forecasting of capacitance loss $(\%)$ at different times $t_{p}$ during the aging time; $t_{p}=[0,24,47,71,94,116,139,149,161,171]$.

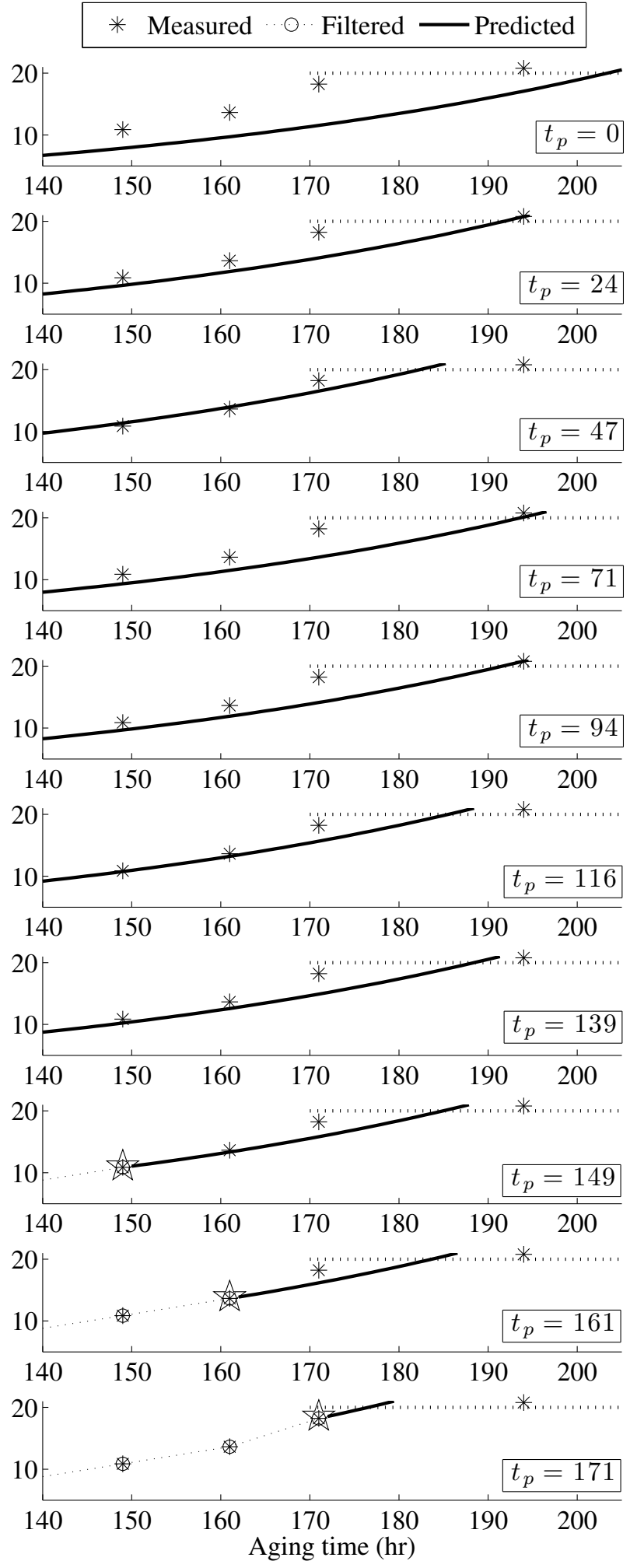

Figure 24. $T_{5}$ : Detail of the health state estimation and forecasting of capacitance loss (\%) at different times $t_{p}$ during the aging time; $t_{p}=[0,24,47,71,94,116,139,149,161,171]$. 


\section{A. Prognostics Alpha-lambda Performance} METRIC

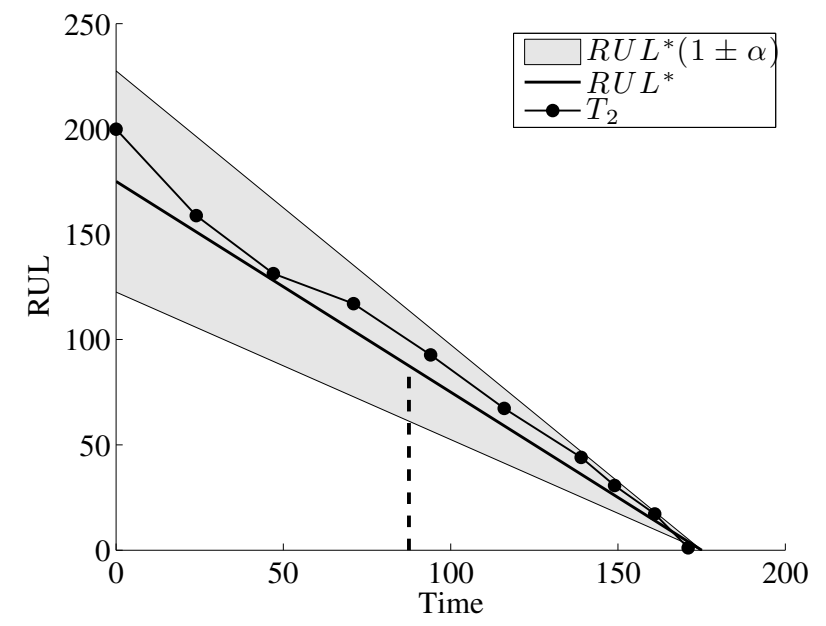

Figure 25. $T_{2}$ : Alpha-Lambda Prognostics Metric $(\lambda=0.5$ and $\alpha=0.3)$.

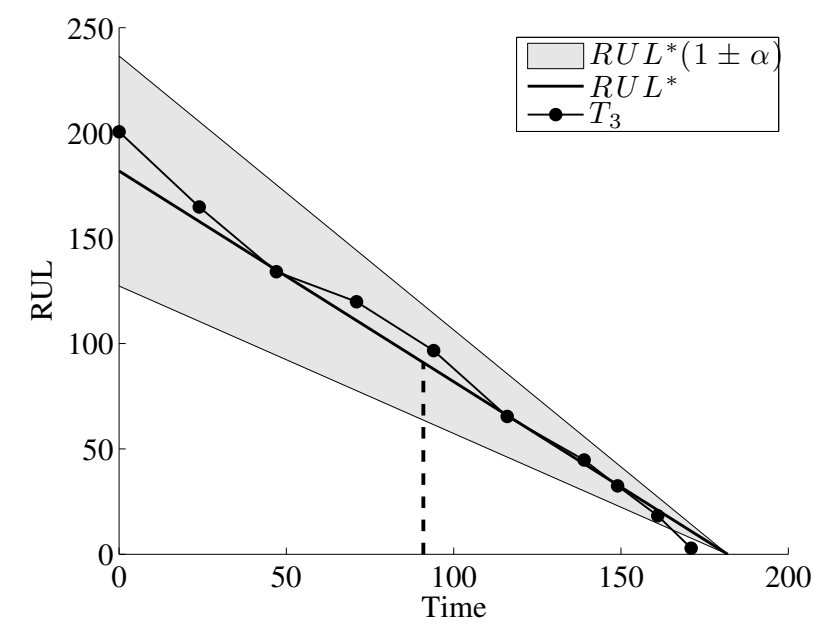

Figure 26. $T_{3}$ : Alpha-Lambda Prognostics Metric $(\lambda=0.5$ and $\alpha=0.3$ ).

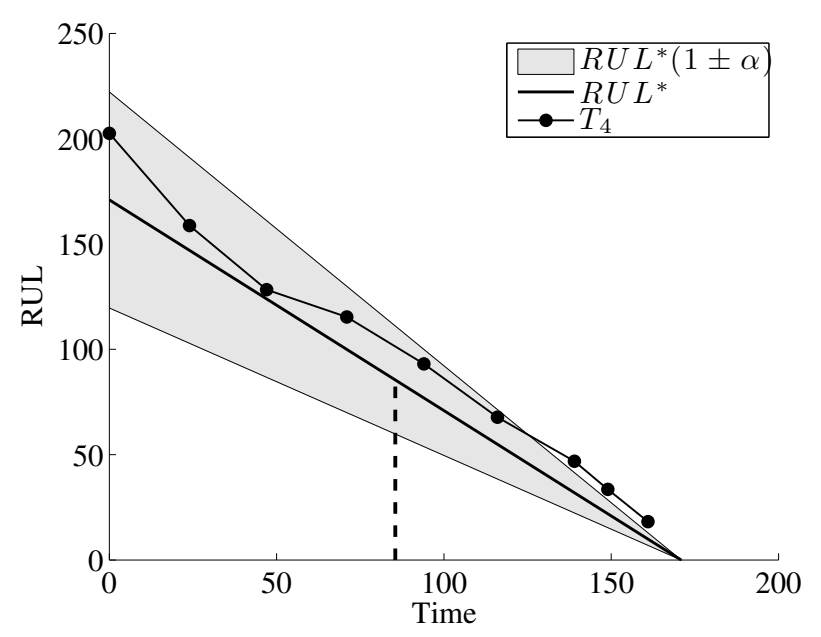

Figure 27. $T_{5}$ : Alpha-Lambda Prognostics Metric $(\lambda=0.5$ and $\alpha=0.3$ ).

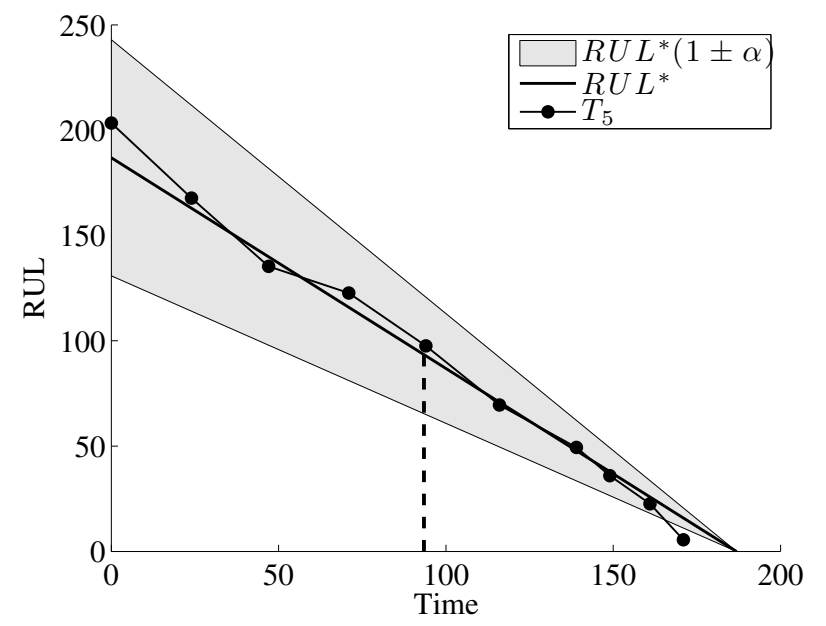

Figure 28. $T_{5}$ : Alpha-Lambda Prognostics Metric $(\lambda=0.5$ and $\alpha=0.3$ ). 